\title{
The Mutual Exclusion Problem
}

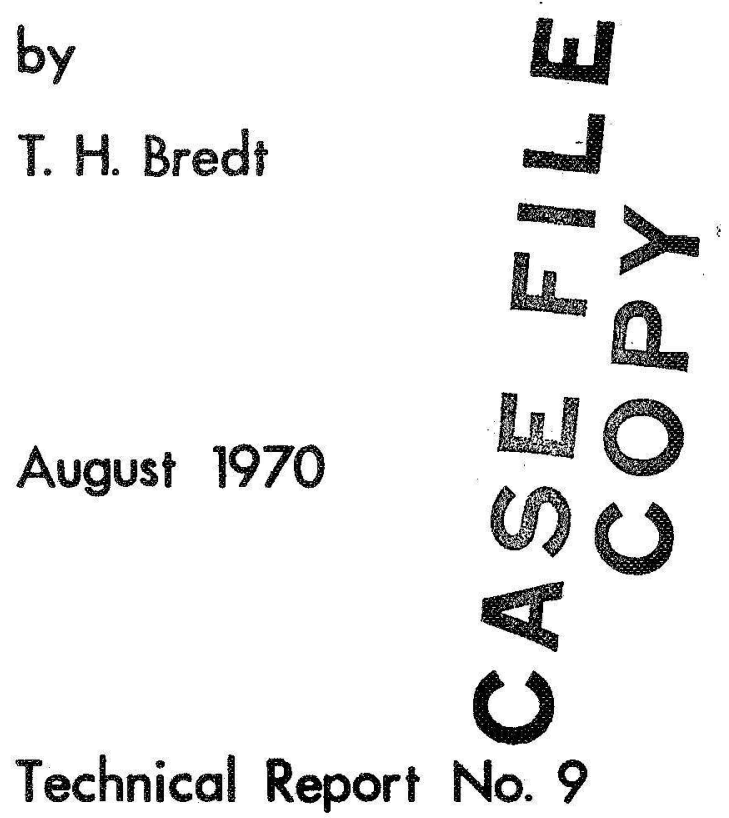

This work was supported in part by the Joint Services Electronic Programs U.S. Army, U.S. Navy, and U.S. Air Force under Contract $\mathrm{N}-00014-67-\mathrm{A}-0112-0044$ and by the National Aeronautics and Space Administration under Grant 05-020-377. 
THE MUTUAL EXCLUSION PROBLEM

by

T. H. Bredt

August 1970

Technical Report No. 9

\section{DIGITAL SYSTEMS LABORATORY}

Stanford Electronics Laboratories Computer Science Department

Stanford University

Stanford, California

This work was supported in part by the Joint Services Electronic Programs U.S. Army, U.S. Navy, and U.S. Air Force under Contract $\mathrm{N}-00014-67-\mathrm{A}-0112-0044$ and by the National Aeronautics and Space Administration under Grant 05-020-337. 
STANFORD UNIVERSITY

Digital Systems Laboratory

Stanford Electronics Laboratories

Computer Science Department

\section{Technical Report Number 9 \\ August , 1970}

THE MUTUAL EXCLUSION PROBLEM

by

T. H. Bredt

\section{ABSTRACT}

This paper discusses how $n$ components, which may be programs or circuits, in a computer system can be controlled so that (1) at most one component may perform a designated "critical" operation at any instant and (2) if one component wants to perform its critical operation, it is eventually allowed to do so. This control problem is known as the mutual exclusion or interlock problem. A summary of the flow table model* for computer systems is given. In this model, a control algorithm is represented by a flow table. The number of internal states in the control flow table is used as a measure of the complexity of control algorithms. A lowex bound of $n+1$ internal states is shown to be necessary if the mutual exclusion problem is to be solved. Procedures to generate control flow tables for the mutual exclusion

* Bredt, T.H. and McCluskey, E.J. A model for parallel computer systems. Technical Report No. 5, SEL Digital Systems Laboratory, Stanford University, Stanfoxd, California (April 1970). 
problem which require the minimum number of internal states are described and it is proved that these procedures give correct control solutions. Other șo-called "unbiased" algorithms are described which require $2 . n$ ! internal states but break ties in the case of mulEiple requests in favor of the component that least recently executed its critical operation. The paper concludes with a discussion of the tradeoffs between central and distributed control algorithms. 
TABLE OF CONTENTS

\begin{abstract}
..............................
TABLE OF CONTENTS ........................

LIST OF TABLES . . . . . . . . . . . . . . . . . . . . iv

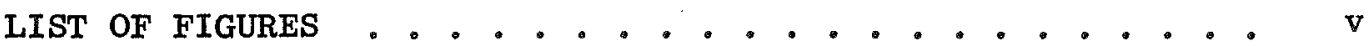

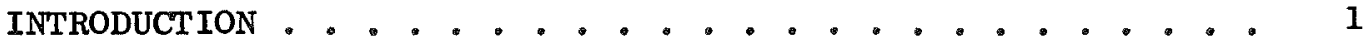

THE FLOW TABLE MODEL FOR PARALIEL SYSTEMS . . . . . . . . 3

A PARALIEL SYSTEM FOR THE MUTUAL EXCLUSTON PROBLEM • . . . 9

CONTROL FLOW TABLES. . . . . . . . . . . . . . . . . 12

Biased Control Flow Tables . . . . . . . . . . . 20

Unbiased Control . . . . . . . . . . . . . 39

CENTRAL VERSUS DISTRIBUTED CONTROL . . . . . . . . . . . . . 49

Linear control . . . . . . . . . . . . . . . 51

Hierarchical Control . . . . . . . . . . . . . 55

CONCLUSIONS . . . . . . . . . . . . . . . . . . . 59

REFERENCES . . . . . . . . . . . . . . . . . . . 60
\end{abstract}




\section{LIST OF TABLES}

1. General Form of a Flow Table ................ . 5

2. Interpretation of Variable Values for Fig. 2......... . 11

3. Sequential Programs for Component $C_{1}$ and a Two-Component Control Mechanism !............ 13

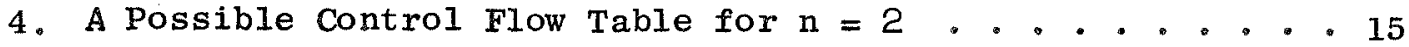

5. Biased Control Flow Tables From Procedure B . . . . . . . 22

6. Possible Entries in Row 0 for $n=3$. . . . . . . . . . . . 29

7. Number of Distinguishable, Correct Flow Tables

With $\mathrm{n}+1$ Internal States................ . 37

8. Flow Table Set-up for $\mathrm{n}=3 . . . . . . . . . . . . .442$

9. Tag for Next Row Given $2,1,3,4$ as Present

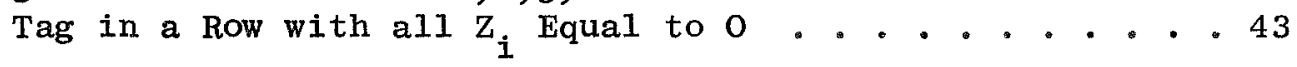

10. Tags for Next Row Given $2,1,3,4$ as Present

Tag and $\mathrm{z}_{2}$ Equal to 1 for the Present Row. . . . . . . . 45

11. Unbiased Control Flow Table From Procedure U, $\mathrm{n}=2$. . . 47

12. Unbiased Control Flow Table From Procedure $U, \mathrm{n}=3 . . . .48$ 
1. Paraliel system for the two-component mutual exclusion problem . . . . . . . . . . . . 6

2. Parallel system for the n-component mutual exclusion problem .................... 10

3. Chains which determine which component to enable for $\mathrm{n}=3 \ldots . . . . . . . . . . . . . .31$

4. Relative chain positions of $i, j$, and $k$. . . . . . . . . 34

5. Incorrect flow table due to the violation of the chain rule $(\mathrm{n}=3), . . . . . . . . . .35$

6. A linear distributed control for the mutual exclusion problem ......... . . . . . . . . . 52

7. General form of a control component for Fig. 6 . . . . . . 53

8. General organization for a linear distributed control . . . 56

9. A binary tree hierarchical control structure . . . . . . 57 
INTRODUCTION

In recent years, there has been a great deal of interest in the mutual exclusion or interlock problem in multiprocessor and multiprogrammed systems $[4,5,6,7,8,9,10,15,16]$. This problem occurs in an environment where several system components (programs or circuits) are operated concurrently. The components are assumed to contain critical operations or instructions (critical sections), whose simultaneous execution must be avoided. Typically, critical sections represent references to a common memory location or possibly the modification of a common system table. A more detailed statement of the mutual exclusion problem is given below.

The Mutual Exclusion Problem:*

Given two or more components in a parallel computer system, which are operated concurrently and contain critical sections, control these components so that the following two restrictions are always satisfied.

Restriction 1:

A most one component is in a critical section at any instant.

Restriction 2:

If a component desires to enter a critical section, it is eventually allowed to do so.

* Dijkstra $[8,9,10]$ has solved a slightly different version of this problem. He did not require that a given program must enter its critical section but rather that the decision as to which program enter its critical section next not be postponed indefinitely. 
For components which are programs, this problem is often solved by defining special hardware instructions and assuming the exclusive execution of these instructions $[7,9,10,15,16]$. In $[4]$ a model for parallel computer systems was proposed in which fundamental-mode flow tables are used to describe the operation of each component. The purpose of this model is to study control problems such as the mutual exclusion problem. In $L 5]$, we have shown how flow tables can be used for the analysis and synthesis of sequential programs. The application of flow tables in the design of sequential circuits is well known $[24]$. As a result, flow tables and the flow table model provide a basis for the study of both hardware and software solutions to the mutual exclusion problem. A flow table solution for the mutual exclusion problem in the case where two components are controlled is given in [4]. This solution was shown to be correct in [ 6 ] using a general analysis method based on the construction of a directed graph representing the state transitions undergone by the entire system. This type of analysis is not feasible as the number of system components becomes large. The notion of correct operation we use is the following. Given a problem, such as the mutual exclusion problem, which is stated in terms of restrictions on system operation, we say that a parallel system is correct with respect to the given problem if the problem restrictions are always satisfied during system operation.

In this paper, the solution of the mutual exclusion problem when arbitrary numbers of processes must be controlled is considered. Several different types of control structures are discussed and 
various solutions or control algorithms given and proved to be correct. These control algorithms are optimal in the sense that they require the minimum number of internal states in a fundamental-mode flow table.

In the next section, a short summary of the flow table model is given. This is followed by the specification of a parallel system for the mutual exclusion problem and the characterization of different control mechanisms.

THE FLOW TABLE MODEL FOR PARALLEL SYSTEMS

A detailed description of this model is given elsewhere $[4,6]$. The essential features are described here. The definition of a parallel system in the flow table model is given below.

Definition 1:

A parallel system is a finite collection of components

$$
C=\left\{c_{1}, c_{2}, \ldots, c_{N}\right\}
$$

and a finite collection of lines

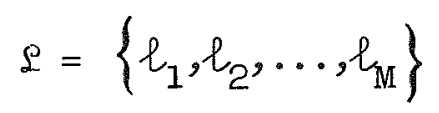

Each component $C_{i}$ has a set of input variables called the input set

$$
\begin{aligned}
& I_{i}=\left\{x_{i_{1}}, x_{i_{2}}, \ldots, x_{i_{m}}\right\} \\
& 1 \leq i_{j} \leq M, j=1, \ldots, m
\end{aligned}
$$


and a set of output variables called the output set

$$
\begin{aligned}
o_{i}=\left\{x_{i_{1}}, x_{i_{2}}, \ldots,\right. & \left.x_{i_{m}}\right\} \\
& 1 \leq i_{j} \leq \mathbb{M}, j=1, \ldots, m
\end{aligned}
$$

Each line $l_{j}=\left(x_{j}, x_{j}\right)$ connects a component output variable $\mathrm{x}_{j}$ with a component input variable $\mathrm{x}_{j}$. The lines carry

level values and value changes propagate from component output to component input. Each output variable must be connected by a line to exactly one input variable and each input variable must be connected by a line to exactly one output variable. The operation of each component is described by a completely specified fundamental-mode flow table with a designated initial internal state. The initial value for each line is the value specified for the output variable associated with the line.

The general form of a fundamental-mode flow table is shown in Table 1. Each row in the flow table represents an internal state of the component whose operation the flow table describes. The present values of the component input and output variables define the component input $\underline{\text { state }}$ and output state, respectively. The total state of a component is defined by its present internal state and input state. The total system state or system state is defined by the $\mathrm{N}$-tuple consisting of the present total state of each of the components. The parallel system designed in [ 4 ] to solve the mutual exclusion problem for two programs is shown in Fig. 1. The initial system state for this parallel system is written $(1-0,1-0,1-00)$. The interpretation of the line values is given in the next section. The initial total state of component $\mathrm{C}_{3}$ 
Table 1. General Form of a Flow Table

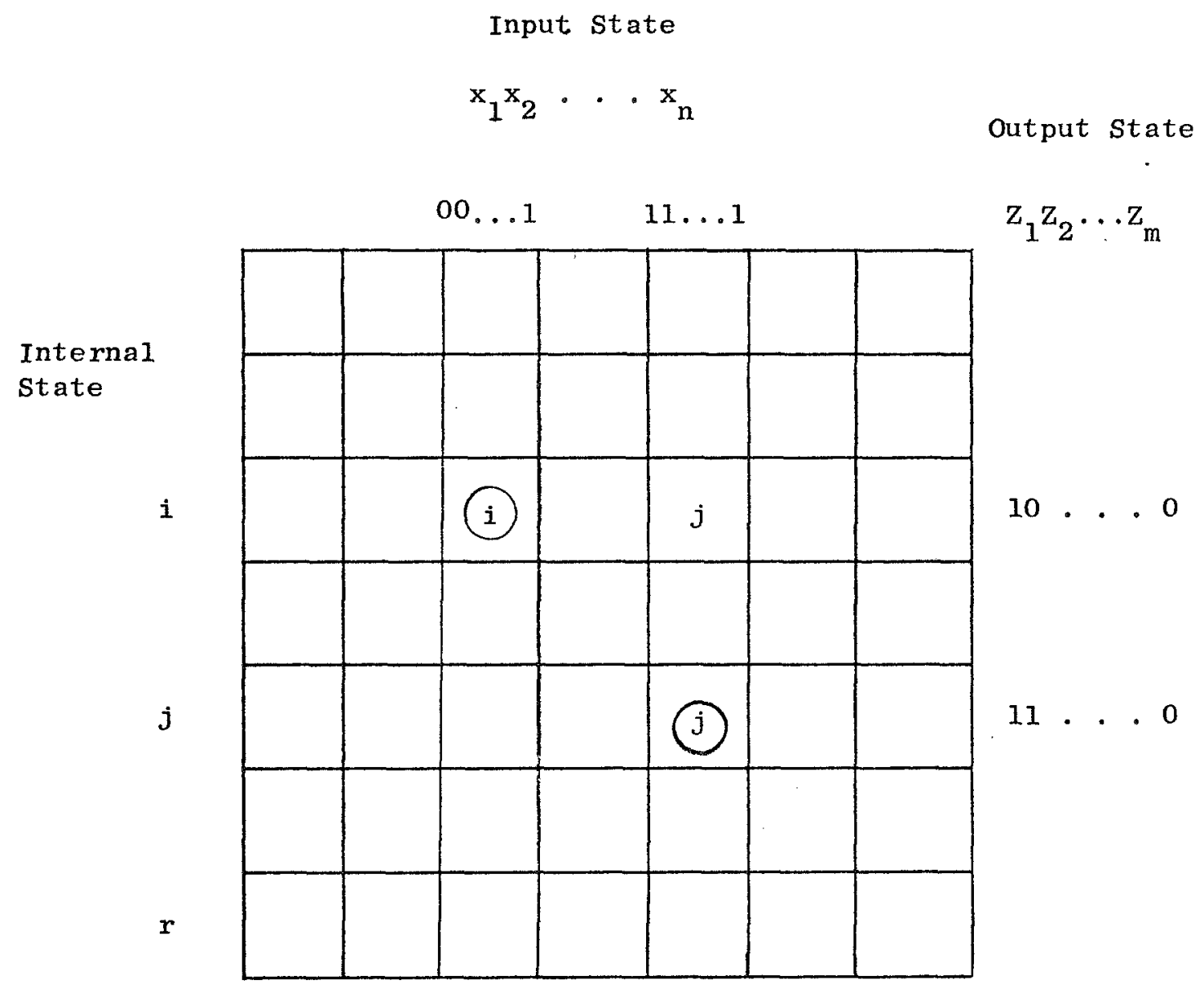

$S$ (next state) 


$$
\begin{aligned}
& c=\left\{\mathrm{c}_{1}, \mathrm{c}_{2}, \mathrm{c}_{3}\right\} \\
& \mathfrak{L}=\left\{l_{1}, l_{2}, l_{3}, l_{4}\right\} \\
& l_{1}=\left(x_{1}, x_{1}\right), l_{2}=\left(x_{2}, x_{2}\right) \\
& l_{3}=\left(z_{1}, z_{1}\right), l_{4}=\left(z_{2}, z_{2}\right) \\
& o_{1}=\left\{x_{1}\right\}, I_{1}=\left\{z_{1}\right\} \\
& o_{2}=\left\{x_{2}\right\}, I_{2}=\left\{z_{2}\right\} \\
& o_{3}=\left\{z_{1}, z_{2}\right\}, I_{3}=\left\{x_{1}, x_{2}\right\}
\end{aligned}
$$
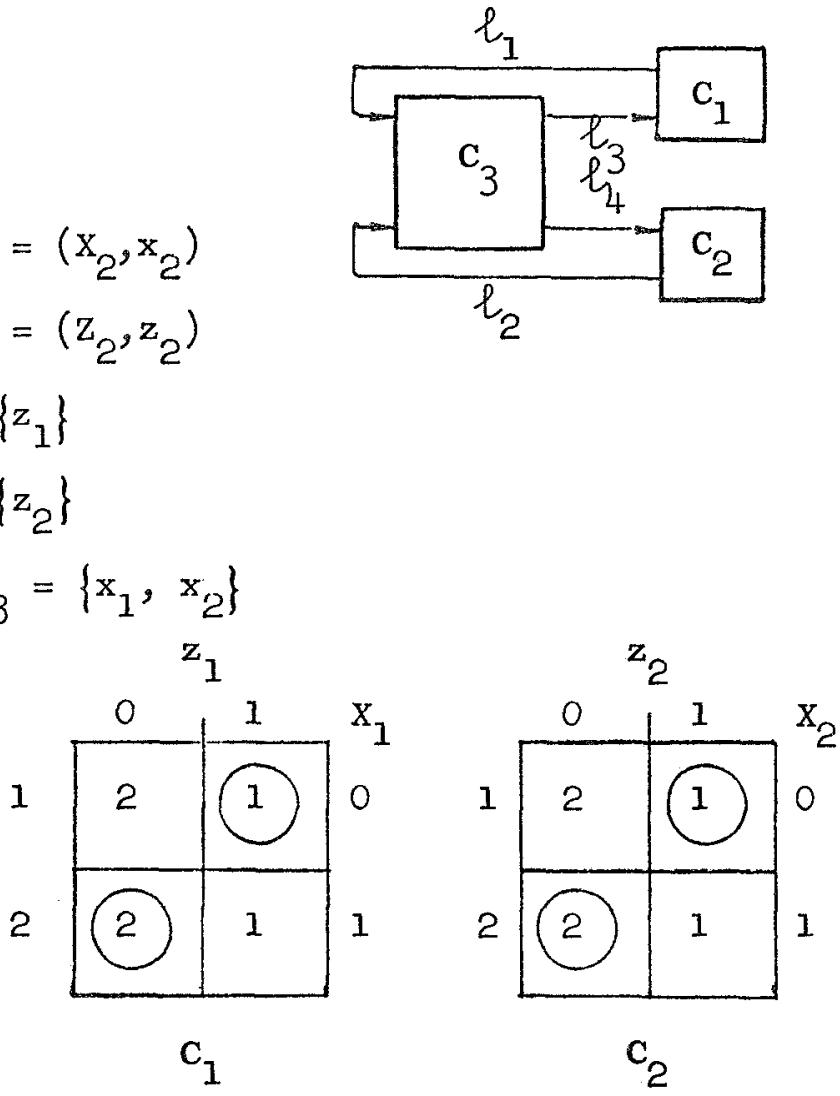

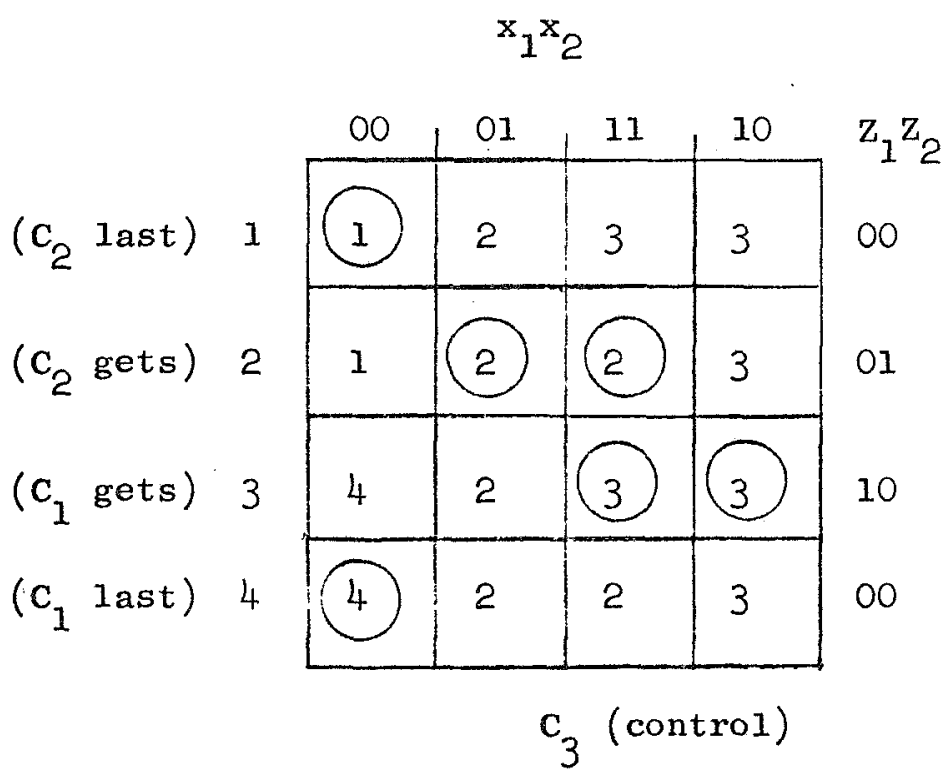

Figure 1. Parallel system for the two-component mutual exclusion problem. 
in Fig. I is written 1-00. If the present internal state is the same as the next-state entry determined by the component total state, the component is said to be stable; otherwise, it is unstable. For a flow table, we require that each unstable entry specifies a stable entry, a table which does not satisfy this condition is called a state table. The assumptions about delays in a parallel system are as follows Assumption 1:

The time for a value change to propagate from a component output to a component input (line delay) is finite and unbounded.

Assumption 2:

Within a component, delays are finite and bounded.

Line delays need not represent "pure" delays and each component is assumed to have no knowledge of the duration of delays in any other component.

The use of flow tables rather than functions to describe component operation distinguishes this model from others $[1,2,3,12,13,14,17,18$, $19,20,21,22,23,25,26,27$ ]. Flow tables provide a direct, formal correspondence between the model and the implementation of the model whether the implementation is a program or a circuit. Our model resembles the model of Muller [25] for speed independent circuits. He restricts components to have single outputs and assumes line delays are zero and component delays are unbounded. In other models components conmunicate by sharing memory cells rather than by wired connections. These models also assume that line delays are zero. The consideration 
of line delays is particularly important in the mutual exclusion problem as is the possibility that two or more components may make simultaneous requests. That is, multiple-input changes may occur at a component. In $[4]$, a mode of operation is defined such that each component input change results in a unique internal-state transition. This mode of operation proceeds in two phases which can be described as follows. When a component enters a stable total state, it determines the present input state by recording in a rank of flip-flops the current input state. This is done using an internal clock signal. The present input state determines whether the component remains stable or undergoes an unstable transition to a new stable internal state. During unstable transitions, computations such as the execution of a critical section may be carried out. This response to the present input state is the second phase of component operation. During this second phase, all input transitions are isolated from the component by the input rank of flip-flops. This two-phase operation defines the basic cycle of operation for a component. We say that a component has recognized an input-variable transition, if the new input value is recorded in an input flip-flop. Because of our line delay assumption, it can be guaranteed that when a component produces an output variable transition, the new value propagates to the input at the other end of the line and is recognized if and only if either the component never changes the output value again or before the output value is changed, the component must recognize an input change produced in recognition of the propagation of its output value to a component input. A further discussion of these considerations is given in [6]. 
A PARALLEL SYSTEM FOR THE MUTUAL EXCLUSION PROBLEM

In the n-process or $\mathrm{n}$-component mutual exclusion problem, there are $\mathrm{n}$ components which contain critical sections. Each component is assumed to enter, leave and then rementer its critical section in an infinite loop. We first consider solutions to the mutual exclusion problem with a single control component or control mechanism. The complete specification of a parallel system, with the exception of the control flow table, for the n-process mutual exclusion problem is Wown in Fig. 2. To clarify the description of the operation of the system, we have deviated slightly from the form specified for a parallel system in Definition 1 in labelling the lines. The interpretation of the variable values for this parallel system is given in Table 2. The operation of each component $c_{i}, 1 \leq i \leq n$, is as follows. Unless specifically stated otherwise, the component is initially in total state 1-0 and is unstable. In this state, the component does not want to enter its critical section and is not in its critical section. Eventually, the component enters total state $2-0$ where $x_{i}$ is set to 1 . The component now wants to enter its critical section and will remain in this total state until it recognizes the enabling value $z_{i}=1$. In total state 2-1 the component enters and leaves its critical section (exactly once). After it has left, the component enters total state 1-1 where $x_{i}$ is set to 0 . This value propagates to the control component which presumably acknowledges the arrival of the $O$ value for $x_{i}$ by setting $z_{i}$ to 0 . When $z_{i}$ becomes 0 , the cycle begins again. 


$$
\begin{aligned}
& e=\left\{c_{1}, c_{2}, \ldots, c_{n+1}\right\} \\
& \mathfrak{L}=\left\{\ell_{1}, \ldots, \ell_{2 n}\right\}
\end{aligned}
$$

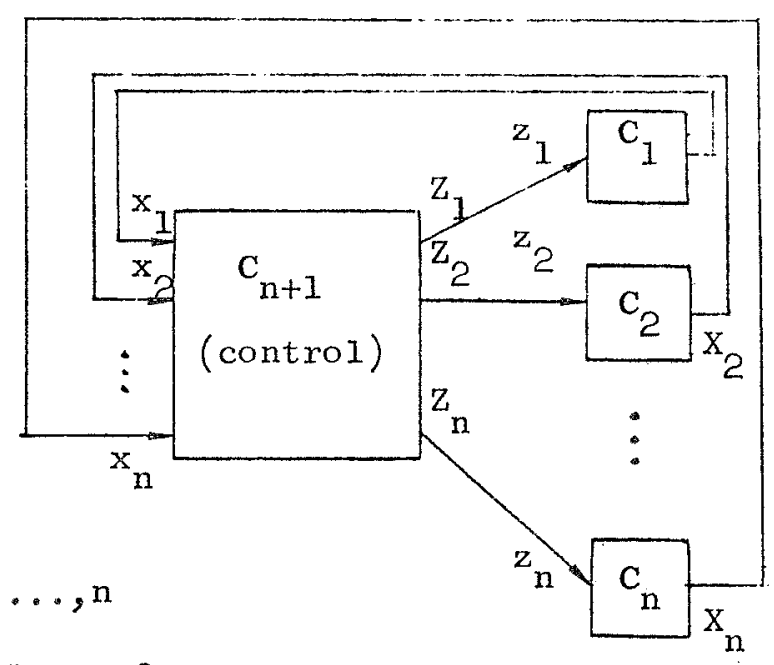

$$
\begin{aligned}
& \ell_{i}=\left(x_{i}, x_{i}\right), i=1, \ldots, n \\
& l_{i}=\left(z_{i}, z_{i}\right), i=n+1, \ldots, 2 n \\
& o_{i}=\left\{x_{i}\right\}, i=1, \ldots, n \\
& I_{i}=\left\{z_{i}\right\}, i=1, \ldots, n \\
& o_{n+1}=\left\{z_{1}, z_{2}, \ldots, z_{n}\right\} \\
& I_{n+1}=\left\{x_{1}, x_{2}, \ldots, x_{n}\right\}
\end{aligned}
$$

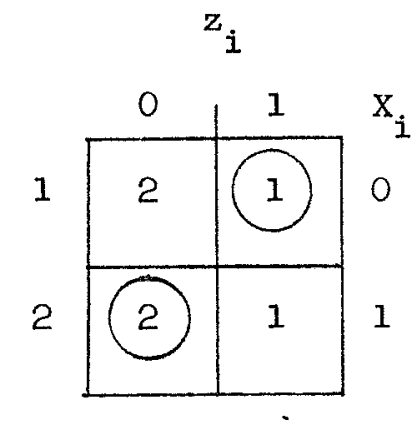

Flow Table for $c_{i}, 1 \leq i \leq n$

Figure 2. Parallel system for the n-component mutual exclusion problem without the control flow table. 
Table 2. Interpretation of Variable Values for Fig. 2

$$
\begin{aligned}
& (1 \leq i \leq n) \\
& x_{i}=0 \quad C_{i} \text { is not in its critical section and does not } \\
& \text { want to enter its critical section. } \\
& x_{i}=1 \quad C_{i} \text { is in its critical section or } C_{i} \text { wants to } \\
& \text { enter its critical section. } \\
& z_{i}=0 \quad C_{i} \text { may not enter its critical section. } \\
& z_{i}=1 \quad C_{i} \text { may enter its critical section. }
\end{aligned}
$$


For two components with critical sections, a control flow table is given in Fig. 1.

In $[5]$, it is shown how to obtain a sequential program from a flow table and how for a certain class of programs, it is always possible to construct a flow table. Program implementations of the flow tables for $\mathrm{C}_{1}$ and a two-process control program are given in Table 3. The assignment statements change values on interconnecting lines. The wait statements are used to test the component input state and to transfer to the appropriate next statement when an input transition occurs. Each pair, e.g. $(0,3)$, specifies an input state and the number of the next statement to be executed if the input state is recognized. There are no restrictions on the exclusive execution of any statements in these programs.

CONTROL FLOW TABLES

In this section, the phrase control flow table refers to a flow table for the control component $\left(\mathrm{C}_{\mathrm{n}+1}\right)$ in Fig. 2. A control flow table is said to be correct if the parallel system of Fig. 2, with that flow table for the control component, is correct with respect to the mutual exclusion problem. We say that a component $C_{i}(1 \leq i \leq n)$ is enabled to enter its critical section when $z_{i}$ has the value 1 . The following definitions serve to partition the class of correct control flow tables. 
Table 3. Sequential Programs for Component $\mathrm{C}_{1}$ and a Two-Component Control Mechanism.

INPUT $\mathrm{Z}_{1}$;

OUTPUT $\mathrm{x}_{1}$; (initially $\mathrm{x}_{1}=0$ )

1: DUMMY; (computation outside critical section)

2: $\quad$ WAIT $(0,3)$;

3: $\quad \mathrm{X}_{1}:=1$;

4: $\quad$ WAIT $(1,5)$;

5: DUMMY; (critical section)

6: $\quad x_{1}:=0$;

7: GO TO 1 .

(a) Program for Component $\mathrm{C}_{1}$

INPUT $\mathrm{x}_{1}, \mathrm{x}_{2}$;

OUTPUT $\mathrm{z}_{1}, \mathrm{z}_{2} ;\left(\right.$ initially $\mathrm{z}_{1}=\mathrm{z}_{2}=0$ )

1: $\mathrm{Z}_{2}:=0$;

2: WAIT $(01,4),(11,7),(10,7)$;

3: $\mathrm{z}_{1}:=0$;

4: $\quad \mathrm{Z}_{2}:=1$;

5: WAIT $(00,1),(10,6)$;

6: $\mathrm{Z}_{2}:=0$

7: $\quad Z_{1}:=1$;

8: WAIT $(00,9),(01,3)$;

9: $\quad \mathrm{z}_{1}:=0$;

10: WAIT $(01,4),(11,4),(10,7)$.

(b) Control Program for Two Components 
Definition 2:

A correct control flow table is said to be unbiased if components are enabled in the order in which their requests are first recognized and if, when multiple requests are recognized simultaneously for the first time, components are enabled in the reverse of the order determined by their most recent access to their critical sections.

The control flow table in Fig. $I$ is unbiased. If a correct control flow table is not unbiased, it is said to be biased. Restriction 2 of the mutual exclusion problem states that if a component $C_{i}(1=\mathbf{i}=n)$ wants to enter its critical section, it is eventually allowed to do so. We will consider this restriction to be violated if it is possible for one or more components to halt outside their critical sections (with $x_{i}$ equal to 0 ) such that some other component, say $\mathrm{C}_{j}$, is prevented from entering its critical section when presumably it wants to do so. For example, the control flow table shown in Table 4 will correctly control two components $\mathrm{C}_{1}$ and $\mathrm{C}_{2}$ in the parallel system of Fig. 2 as long as both $\mathrm{C}_{1}$ and $\mathrm{C}_{2}$ run forever. The initial system state with this control flow table is $(1-1,1-0,1-00)$. Component $C_{1}$ is assumed to have just left its critical section and will not be permitted to enter again until after $\mathrm{C}_{2}$ is enabled. If $\mathrm{C}_{2}$ should halt, $\mathrm{C}_{1}$ will never be enabled again. Dijkstra $[8,9,10]$ also does not allow control solutions which fail if one or more programs halt. 
Table 4. A Possible Control Flow Table for $\mathrm{n}=2$

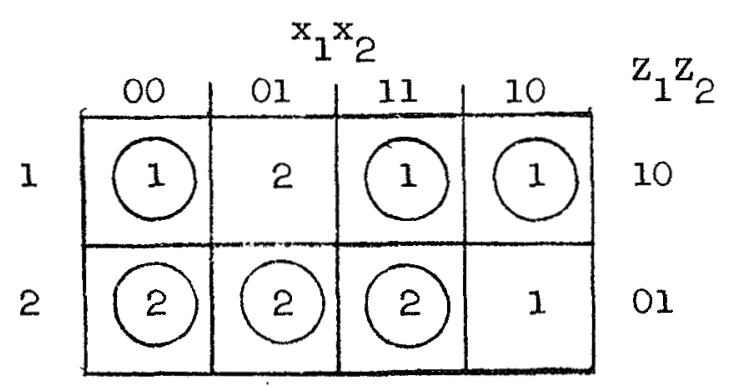


The following theorems establish necessary conditions for correct control flow tables.

Theorem 1:

If a control flow table is correct, there must be at least one internal state with an output state for which $\mathrm{Z}_{i}$ has the value $1, i=1, \ldots, n$.

Proof:

If there is no output state for which $Z_{i}$ has the value 1 , then $z_{i}$, the input to component $C_{i}$, will never equal 1 . Therefore component $C_{i}$, will never enter its critical section violating Restriction 2 of the mutual exclusion problem.

Theorem 2:

The output state for the initial total state of a correct control flow table must have $Z_{i}$ equal to 0 for all $i, i=1, \ldots, n$.

Proof:

Suppose there exists a correct control flow table with $\mathrm{Z}_{i}$ equal to 1 for some $i$ in the initial total state. Initially, lines are assumed to be stable and components $C_{i}$ are assumed to be in internal state 1 . Thus component $C_{i}$ is in the stable total state 1-1. Component $C_{i}$ may not request to enter its critical section until $z_{i}$ becomes 0 . The control should be stable initially since all $x_{i}$ are 0 . The control will not 
leave its stable initial state until a different input state is recognized. Since $x_{i}$ cannot become 1 , some other component must request access to a critical section before $z_{i}$ can be changed to 0 . This is not allowed in a correct control flow table.

Theorem 3:

If a control flow table is correct, $Z_{i}$ is changed from 0 to 1 only if $x_{i}$ is 1 .

Proof: (By contradiction)

Suppose $z_{i}$ is changed from 0 to 1 when $x_{i}$ is 0 . The output variable $x_{i}$ of component $c_{i}$ may be 0 or 1 . If $x_{i}$ is 0 , component $C_{i}$ could be trapped in total state $1-1$ and either never released or released only after a transition for another control input. Neither case is allowed for a correct control flow table. If $\mathrm{x}_{i}$ is $1, \mathrm{C}_{i}$ is in internal state 2 . As soon as $z_{i}$ becomes 1 , $C_{i}$ may enter its critical section. It is possible that before the input variable $x_{i}$ becomes 1 , a different input, say $x_{j}$, becomes 1 . If the control does not set $z_{j}$ to 1 until after $x_{i}$ becomes 1 then the enabling of $C_{j}$ depends on the operation of $C_{i}$ which is not allowed. If $\mathrm{Z}_{j}$ is set to 1 before $x_{i}$ becomes 1 , both $\mathrm{C}_{i}$ and $\mathrm{C}_{j}$ may enter their critical sections simultaneously which violates Restriction 1 and the control flow table is not correct. 
Theorem 4:

If a control flow table is correct, it must never enter a total state for which the output state has more than one output variable with the value 1 .

Proof:

Suppose such a total state is entered and $Z_{i}$ and $z_{j}$ have the value 1 . By Theorem $3, x_{i}$ and $x_{j}$ must be equal to 1 . It is possible that $x_{i}$ and $x_{j}$ are also 1 since $x_{i}$ and $x_{j}$ are not set to 0 until the inputs $z_{i}$ and $z_{j}$ have the value 1 and line delays cannot be controlled. Therefore $C_{i}$ and $C_{j}$ may both be in internal state 2 and may simultaneously enter their critical sections. This violates Restriction 1 and the control flow table cannot be correct.

Theorems 1-4 enable us to prove the following theorem which establishes a lower bound on the number of internal states required for a correct control flow table.

Theorem 5:

For a given $n$, every correct control flow table must have at least $n+1$ internal states.

Proof :

By Theorem 2, the output state for the initial internal state must have $z_{i}$ equal to 0 for all $i, i=1, \ldots, n$. By Theorems 1 and 4 , there must be at least one internal state for which $z_{i}$ has the value 1 and $z_{j}$ has the value $0, j \neq i$ for each $i, i=1, \ldots n$. 
The number of internal states required by a correct control flow table provides a measure of the complexity or cost of the control function or algorithm for the mutual exclusion problem which is independent of whether the algorithm is implemented as a program or as a circuit.

The following theorem establishes another necessary condition for a correct control flow table. This condition is not required to determine the lower bound on the number of internal states but will be used later.

Theorem 6:

If a control flow table is correct, output variable $z_{i}$ is changed from 1 to $O$ only if $x_{i}$ is 0 .

Proof:

Suppose $z_{i}$ is changed from 1 to 0 when $x_{i}$ is 1 . By Theorems 2 and 4 either the output state for the next internal state has no $z_{j}$ equal to $I$ or exactly one $z_{j}$ equal to 1 . Suppose no $z_{j}$ is equal to 1 . Now if some $x_{k}$ becomes 1 , it must be recognized in a finite time (Assumption 2) and the control flow table must enter a stable state for which $z_{k}$ is 1 . If it does not, the enabling of $C_{k}$ depends on the activity of some other component, which is forbidden. The enabling of $\mathrm{C}_{k}$, allows $\mathrm{C}_{k}$ to enter its critical section. During this interval, from the moment the state for which $z_{i}$ is 1 is left until $c_{k}$ enters its critical section, the 1 value produced on $z_{i}$ may be propagating to the input of 


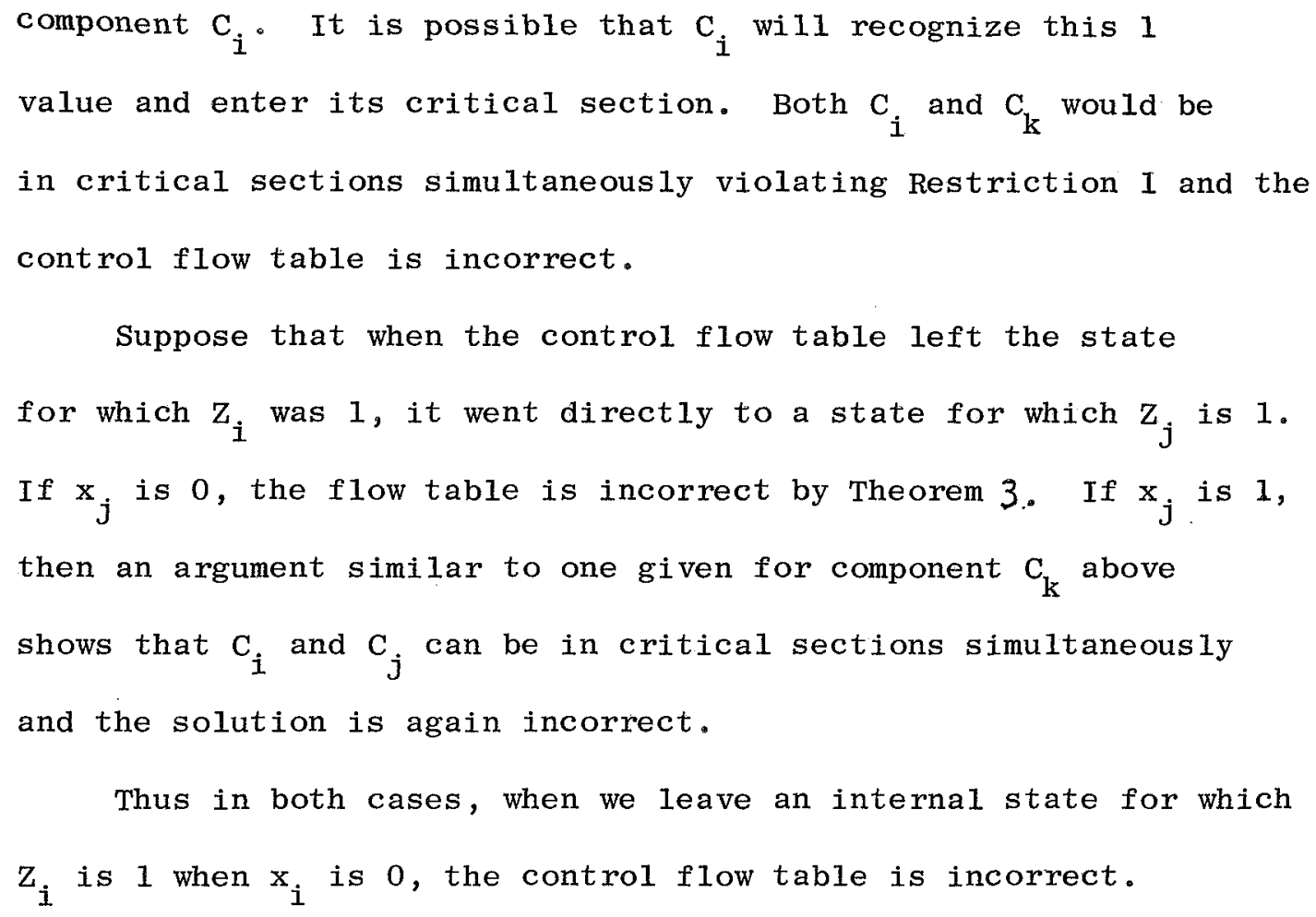

For a given value of $n$, the following procedure constructs a biased control flow table with the minimum number of internal states.

\section{$\underline{\text { Procedure B }}$}

(Biased Control Flow Table With $\mathrm{n}+1$ Internal States)

1. Define a flow table with $2^{\mathrm{n}}$ columns, one column for each possible input state, and $n+1$ internal states (rows), which are numbered $0,1,2, \ldots, n$.

2. Let the initial internal state be state 0 with output state such that $z_{i}$ has the value 0 for all $i$.

3. Let $Z_{i}$ have the value 1 and $z_{j}$ the value $0(j \neq i)$ in the output state for row i. 
4. Define the table entries as follows.

a. In row 0

In each column, the entry is the same as the least subscript of an input variable with the value 1 . The entry is 0 if all $x_{i}$ are 0 .

b. In row $i(1 \leq i \leq n)$

In each column, if $x_{i}$ has the value 1 , the entry is (i). If $x_{i}$ has the value 0 , the entry is the least $j$ such that $j>i$ and $x_{j}$ has the value 1 or, if no such $j$ exists, the least $j$ such that $x_{j}$ has the value 1 . If all input variables are 0 , the entry is 0 .

The flow tables generated by Procedure $B$ for $n=2$ and $n=3$ are shown in Table 5. These flow tables are biased because, in the case of a particular multiple request, in each row the same component is enabled regardless of which component last executed a critical section.

Theorem 7:

For each $n$, the flow table obtained from Procedure $B$ is correct.

The proof of Theorem 7 will be a direct consequence of a later theorem. 
Table 5. Biased Control Flow Tables From Procedure B

\begin{tabular}{|c|c|c|c|c|}
\hline \multicolumn{5}{|c|}{$x_{1} x_{2}$} \\
\hline 00 & 01 & 11 & 10 & $\mathrm{z}_{1} \mathrm{z}_{2}$ \\
\hline (0) & 2 & 1 & 1 & 00 \\
\hline 0 & 2 & 1) & (1) & 10 \\
\hline 0 & (2) & (2) & 1 & 01 \\
\hline
\end{tabular}

(a) $n=2$

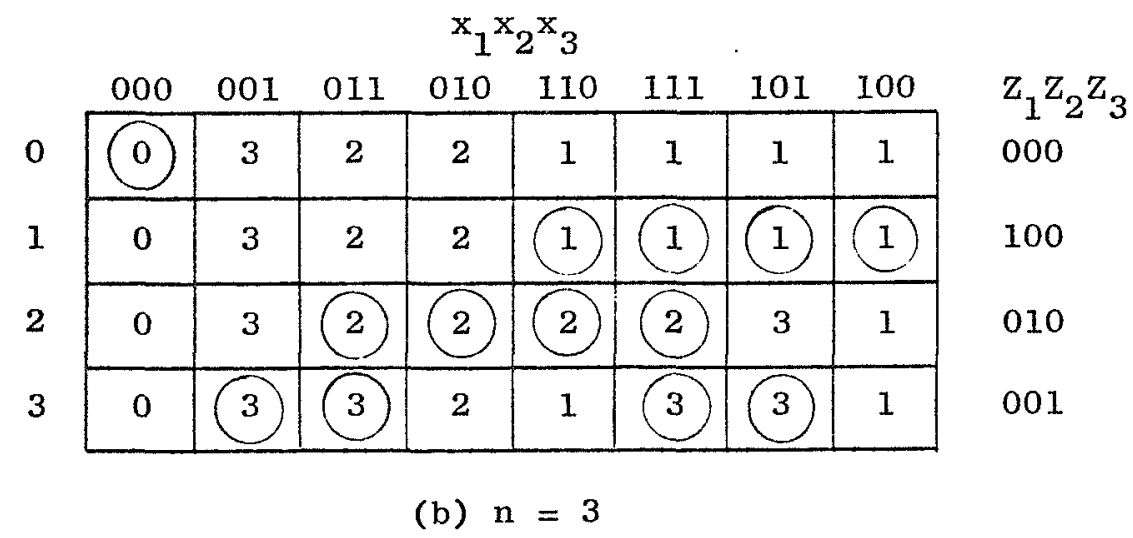


With the control algorithm defined by the control flow table, a component must wait for at most n-1 other components to execute critical sections before it executes its own critical section. We will now consider the general class of biased control flow tables which are correct and use the minimum number of internal states.

Definition 3:

Two flow tables are said to be distinguishable if when the same input sequence is recognized by each flow table, different output sequences are produced.

If two flow tables are not distinguishable, they are indistinguishable. We will determine the number of distinguishable control flow tables that are correct and have $n+I$ internal states. Next we give conditions sufficient to guarantee that Restriction 1 of the mutual exclusion problem is satisfied.

Theorem 8:

If a control flow table is such that the following conditions hold:

1. The flow table is initially stable with initial output state in which $z_{i}$ is equal to 0 for all $i, i=1, \ldots, n$.

2. Each output state has at most one $z_{i}$ with the value 1 . 
3. The value of output variable $z_{i}$ is changed from $O$ to 1 only if $x_{i}$ is 1 .

4. The value of output variable $z_{i}$ is changed from 1 to 0 only if $x_{i}$ is $O$.

Then, at most one component $c_{i}(1 \leq i \leq n)$ may be in a critical section at any instant (Restriction 1 is satisfied).

Proof:

By condition 1 and the definition of the parallel system in Fig. 2, no component is initially in its critical section. By condition 3 and the flow table specification of operation for component $\mathrm{c}_{\mathbf{i}}(1 \leq \mathrm{i} \leq \mathrm{n})$ in Fig. 2 , a component is enabled to enter its critical section $\left(z_{i}\right.$ is set to 1$)$ only if that component is in the stable total state $2-1$. If $z_{i}$ is set to 1 , condition 4 ensures that it is not set to $O$ until $x_{i}$ becomes $O$. But the flow table for component $C_{i}$ shows that $x_{i}$ cannot become $O$ until after $C_{i}$ has left its critical section and entered the stable total state 1-1. Component $C_{i}$ cannot re-enter its critical section until $z_{i}$ becomes 0 , which only happens after $x_{i}$ becomes $O$ at the control input. It follows that if component $c_{i}$ is in its critical section, then $z_{i}$ must have the value 1 or, equivalently, if $z_{i}$ has the value 0 , component $C_{i}$ is not in its critical section. The fact that at most one component may be in a critical section at any instant follows from condition 2. 
The following theorem establishes the output state requirements for a correct control flow table with $n+1$ internal states.

Theorem 9:

If a control flow table is correct and has $n+1$ internal states, then

1. The output state for the initial internal state must have $z_{i}$ equal to 0 for all $i, i=1, \ldots, n$.

2. For each $i, i=1, \ldots, n$, there must be exactly one output state with $z_{i}$ equal to $I$ and $z_{j}$ equal to 0 for all $j, j \neq i$.

Proof:

If condition 1 does not hold, the flow table is incorrect by Theorem 2. If condition 2 does not hold, then either some $z_{i}$ is never equal to 1 in any output state, which is not allowed by Theorem 1 , or more than one $Z_{i}$ is 1 in some output state, which is not allowed by Theorem 4 .

In the remainder of this section the phrase "control flow table" refers to a control flow table with $n+1$ internal states, numbered $0,1, \ldots, n$, and $2^{n}$ columns. The initial internal state is state 0 with output state in which $z_{i}$ is 0 for all $i$. The output state for row $i$, $i=1, \ldots, n_{\text {, }}$ is $z_{i}$ equal to $I$ and $z_{j}$ equal to $0, j \neq i$. 
The selection of table entries in row $O$ is covered by the following two theorems.

Theorem 10:

Consider a control flow table with $n+1$ internal states as just defined. If this flow table is correct, the entries in row $O$ must satisfy the following conditions.

1. In the column in which all input variables have the value 0 , the entry is 0 .

2. In the other columns, the entry is $j$ where $j$ is the subscript of an input variable $x_{j}$ that has the value 1 in that column.

Proof:

If condition $I$ does not hold, some $z_{i}$ is set to $I$ when $x_{i}$ is 0. By Theorem 3, the flow table is incorrect. If condition 2 does not hold, either the entry is 0 , a stable entry, or the entry specifies a row with $z_{i}$ equal to 1 in a column with $x_{i}$ equal to 0 . The latter case is ruled out by Theorem 3 . If the entry is $O$, the enabling of a component depends on an input change produced by another component, which is not allowed. 
Theorem 11:

Consider a control flow table with $n+1$ internal states as defined earlier. If the entries in rows 1 through $n$ are specified correctly and

1. In the column with all $x_{i}$ equal to 0 , the entry is 0 .

2. In the other columns, the entry is $j$ where $j$ is the subscript of an input variable $x_{j}$ that has the value 1 in that column.

then the flow table is correct and each choice of the entries in row 0 results in a distinguishable control flow table.

Proof:

Each choice of an entry in a column with some $x_{i}$ equal to 1 specifies an internal state with a different output state; therefore, each choice of the row 0 entries results in a distinguishable flow table. The correctness of the flow table follows from the fact that when a 1 input value is recognized, exactly one of the components which produced a input value is enabled and also from the assumption that rows 1 through $n$ are correctly specified.

There are $2^{n}-(n+1)$ entries in row 0 in columns where more than one input variable has the value 1 ( $n$ entries have exactly one input variable equal to 1 and one has no input variables equal to 1 ). 
There are $\left(\begin{array}{l}n \\ p\end{array}\right)$ entries with exactly $p$ input variables equal to $1^{*}$. By Theorem 9, for each of these entries there are p ways to select that entry and each selection gives a distinguishable flow table, assuming rows 1 through $\mathrm{n}$ are correct. Therefore, the total number of distinguishable flow tables which can be produced on the basis of row 0 alone is

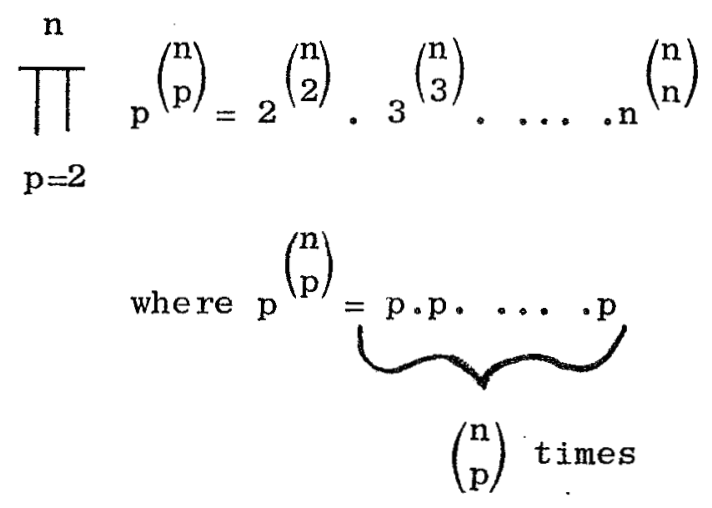

For $n=3$, Table 6 shows the possible entries in row 0.

$*\left(\begin{array}{l}n \\ p\end{array}\right)=\frac{n !}{p !(n-p) !}$ 
Table 6. Possible Entries in kow 0 for $\mathrm{n}=3$

\begin{tabular}{|c|c|c|c|c|c|c|c|}
\hline 000 & 001 & 011 & 010 & 110 & 111 & 101 & 100 \\
\hline (0) & 3 & 2,3 & 2 & 1,2 & $1,2,3$ & 1,3 & 1 \\
\hline
\end{tabular}

$$
\begin{aligned}
\prod_{\mathrm{p}=2}^{3} \mathrm{p}^{\left(\begin{array}{l}
3 \\
\mathrm{p}
\end{array}\right)}=2^{\left(\begin{array}{l}
3 \\
2
\end{array}\right)} \cdot 3^{\left(\begin{array}{l}
3 \\
3
\end{array}\right)} \\
=2^{3} \cdot 3=24
\end{aligned}
$$


It remains to consider how the entries in rows 1 through n may be chosen to give correct, distinguishable flow tables. The flow tables produced by Procedure B use a fixed "rule" to determine, in the case of multiple requests, which component is to be enabled next. This rule is stated in step $4 \mathrm{~b}$ of the procedure. This rule can be restated in more general terms. For each $n$, there is a "chain" consisting of a circular ordering of the $n$ integers $1,2, \ldots, n$. In the case of Procedure $B$, the next integer after integer $i$ in the chain is given by the sum $i(\bmod n)+1$. The chain for $n=3$ is shown in Fig. 3a. The rule to determine the entries in row $i$ can be restated in terms of the "chain rule" below.

Chain Rule:

Consider row $i(1 \leq i \leq n)$. The next-state entry in each column is speaified by the next integer $j$ in the chain after or including the integer $i$ such that $x_{j}$ has the value 1 in that column. If all input variables have the value 0 , the entry is 0 .

The chain of Fig. $3 a$ and the chain rule produce the same next-state entries for $n=3$ as the Procedure $B$. For $n=3$, there is one other chain that is different from the chain in Fig. 3a. This chain is shown in Fig. 3b. In general, for each $n$, there are $(n-1)$ : distinct chains since the present position is always fixed by the row number and there are $(n-1)$ : possible arrangements of the other $n-1$ integers. 


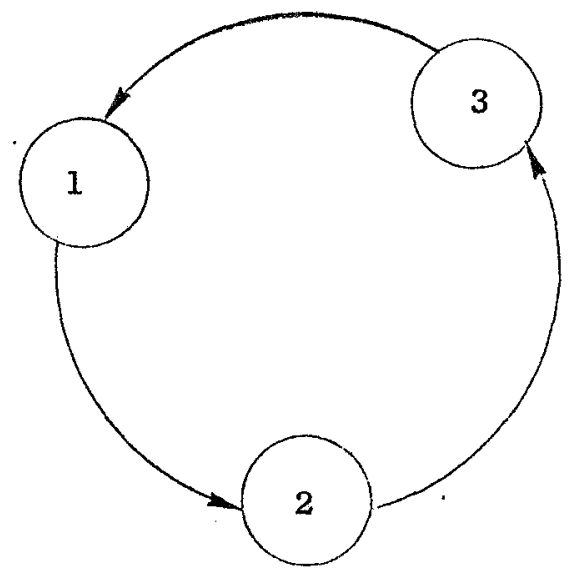

(a) Chain used in Procedure B

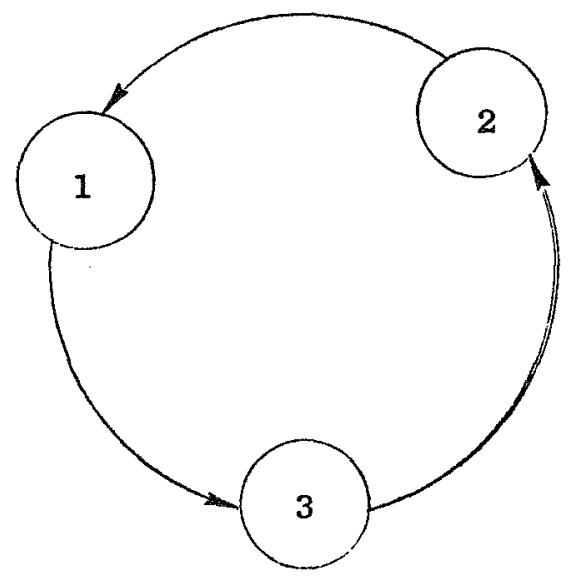

(b) Another chain

Figure 3. Chains which determine which component to enable for $\mathrm{n}=3$. 
The importance of chains and the chain rule in the determination of the entries in rows 1 through $\mathrm{n}$ is demonstrated by the following two theorems.

Theorem 12:

Assume the entries in row $O$ are chosen correctly. If the selection of entries in rows 1 through $n$ of a control flow table with $n+1$ internal states as defined earlier does not follow the chain rule for any chain, the flow table is incorrect.

\section{Proof:}

There are two ways to violate the chain rule. One way is to have a non-zero entry in the column with all input variables equal to 0 . As a result, some output variable $Z_{i}$ is set to 1 when $x_{i}$ is $O$ and the flow table is incorrect by Theorem 3 . The other way involves the selection of next-state entries in columns with at least one input variable equal to 1 . Suppose the rule is violated in the case exactly one input variable is equal to 1. Then the flow table is incorrect by Theorem 3. Next, consider the violation of the chain rule when more than one input variable has the value 1. If, in row $i$, the violation occurs such that row $i$ is left when $x_{i}$ has the value 1 , the flow table is incorrect by Theorem 6. Suppose this is not the case. There must be an entry in some row, say row i, such that for some input state with at. least two input variables, $x_{j}$ and $x_{k}(j, k \neq i)$ equal to 1 , the 
rule specifies that the entry should be $j$ and the entry is $k$ instead. Thus, component $C_{k}$ enters its critical section next instead of $\mathrm{C}_{j}$. Suppose that, while in row $k$, input variable $x_{i}$ becomes equal to 1 again (component $C_{i}$ wants to re-enter its critical section). When $x_{k}$ becomes 0 , both $x_{i}$ and $x_{j}$ are equal to 1 along with all other input variables that were equal to 1 in row $i$ or became equal to 1 while the control component was in internal state $k$. The relative positions of $i, j$, and $k$ in the chain are shown in Fig. 4. From row $k$, component $c_{i}$ must be enabled (row $i$ must be entered) before component $C_{j}$ is enabled. Otherwise, the chain rule is not violated. Suppose that while in row $i$ and when $x_{i}$ becomes 0 , the input state recognized is exactly the same as the previous time the flow table was in row $i$. As before, row $\mathrm{k}$ is entered next. For this pattern of requests, row $j$ is never entered and component $c_{j}$ never enters its critical section. Restriction 2 is violated and the flow table is incorrect.

Theorem 12 is equivalent to saying that if the entries in row 0 are specified correctly and the flow table is correct, then the entries in rows 1 through $n$ must satisfy the chain rule for some chain. This establishes the necessity for using the chain rule. An example of the violation of the chain rule for all chains is shown in Fig. 5. The 2-101 entry is incorrect for the chain of Fig. 3a (it should be 3). For the chain of Fig. 3b, the 1-01l entry should be 3 and the 3-110 entry should be 2. The undesired transitions are also shown in Fig. 5 . 


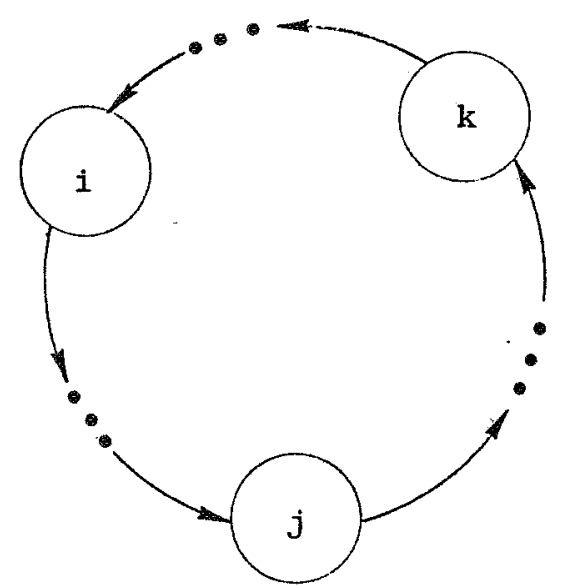

Figure 4. Relative chain positions of $i, j$, and $k$. 


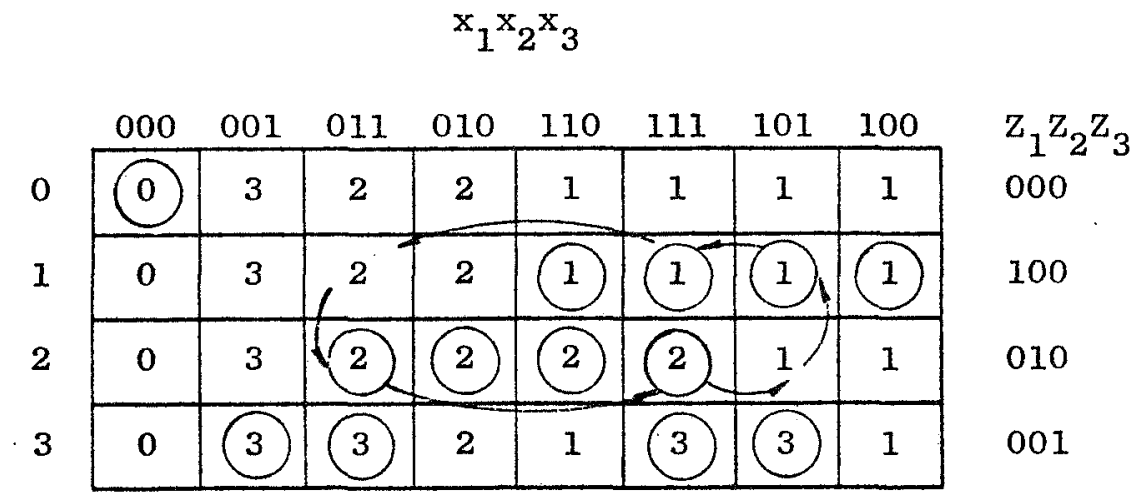

Figure 5. Incorrect flow table due to the violation of the chain rule $(n=3)$. 
Either $i=2, j=3$, and $k=1$ with initial input state 111 or $i=1$, $\mathrm{j}=3$, and $\mathrm{k}=2 \mathrm{with}$ initial input state 111 .

No two chains result in exactly the same specification of nextstate entries and each assignment gives a distinguishable flow table for some input sequence. Therefore the total number of distinguishable, correct flow tables which have $n+1$ internal states is

$$
(n-1) ! \prod_{p=2}^{n} p^{\left(\begin{array}{l}
n \\
p
\end{array}\right)}
$$

The values of this expression for $n=2,3$, and 4 are given in Table 8 . The following theorem establishes that following the chain rule with some chain to fill in the entries in rows 1 through $n$ is sufficient to solve the mutual exclusion problem if the entries in row 0 are specified correctly.

Theorem 13:

Given a control flow table with $\mathrm{n}+1$ internal states as described earlier. If the entries in row $O$ are chosen correctly and the entries in rows 1 through $n$ are chosen using the chain rule with a fixed chain, the flow table is correct. 
Table 7. Number of Distinguishable, Correct Flow Tables With $n+1$ Internal states

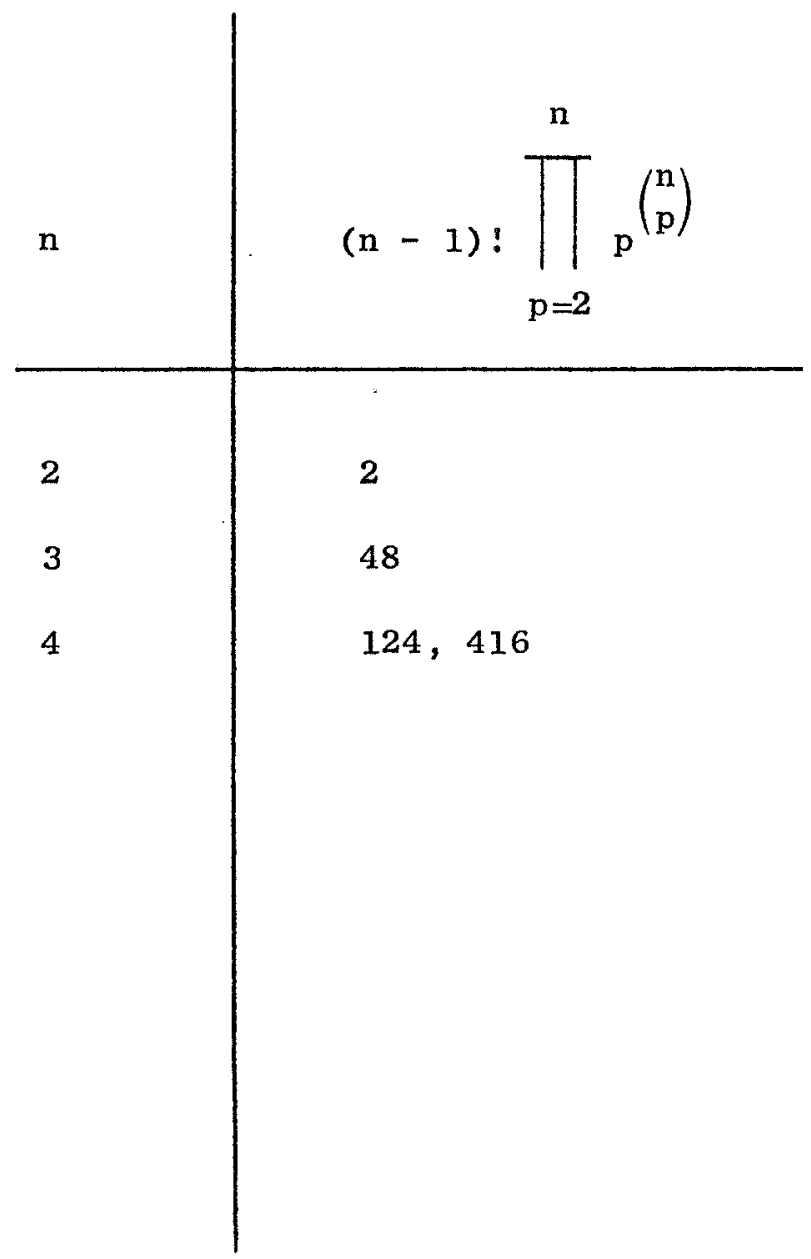


Proof:

The flow table definition and the entries determined by the chain rule satisfy conditions 1 - 4 of Theorem 8; therefore, Restriction 1 of the mutual exclusion problem is satisfied. Suppose Restriction 2 can be violated. That is, some component $C_{i}$ is such that $x_{i}$ is $I$ but $z_{i}$ is never set to 1 . If there are not multiple requests (more than one input variable is 1 ), $C_{i}$ will be enabled. Therefore more than one input variable must be 1. But, the definition of the chain rule and the structure of a chain guarantee that after at most $n-1$ components enter their critical sections, component $C_{i}$ must be enabled.

Combining the results of Theorems $10-13$, we have the following theorem which establishes necessary and sufficient conditions for a correct control flow table with $\mathrm{n}+1$ internal states.

Theorem 14:

Consider a control flow table with $n+1$ internal states as defined earlier. This flow table is correct if and only if

1. In row 0

a. In the column with all $x_{i}$ equal to 0 , the entry is 0 .

b. In the other columns, the entry is $j$ where $j$ is the subscript of an input variable $x_{j}$ that has the value 1 in that column. 


\section{In rows 1 through $n$, the entries are chosen by using the chain rule with a fixed chain.}

The proof of Theorem 7 follows as a corollary of Theorem 14. Notice that Theorem 14 defines a general procedure which can be used to construct a correct flow table with $n+1$ internal states. This procedure can be used instead of the Procedure B defined earlier.

This completes our discussion of control flow tables that have the minimum number of internal states.

Unbiased Control Flow Tables

Let us consider unbiased control flow tables (Definition 2). By Theorem 2, there must be at least one row with output state for which $z_{i}$ is 0 for a.l $i, i=1, \ldots, n$. By Theorem 1 , there must be at least one row for which $z_{i}$ is 1 and $z_{j}$ is 0 for all $j, j \neq i$, $1 \leq i, j \leq n$. There must be $n$ : rows with all output variables equal to 0 . Each row must correspond to a unique past history of critical section executions. This must be done in order to 
ensure that when multiple simultaneous requests are recognized, the component enabled is the component which has been out of its critical section the longest. There must be $(n-1) !$ rows with exactly one $z_{i}$ equal to 1 for each $i, i=1, \ldots, n$. This is also required to decide which component to enable when component $C_{i}$ leaves its critical section. Thus in order to be unbiased, the control flow table must have "perfect memory". The total number of internal states is $n:+n(n-1) !=2 n ! . \quad$ The following theorem has been established.

Theorem 15:

For a given $n$, an unbiased control flow table must have at least $2 n$ ! internal states.

Theorem 5 and Theorem 15 show that the cost, in terms of the number of internal states, of providing unbiased service is rather high. The following procedure can be used to construct an unbiased control flow table for any specified value of $n$.

\section{Procedure U}

(Unbiased Control Flow Table With 2n! Internal States)

1. Define a flow table with $2^{n}$ columns and $2 n$ : internal states.

2. Let the first $n$ : internal states (rows) have the output state with all $Z_{i}$ equal to 0 . Give each of these rows a unique tag which is one possible order of the subscripts of the $n$ components $\mathrm{C}_{1}, \ldots, \mathrm{c}_{\mathrm{n}} *$.

\footnotetext{
* We adopt the convention that the leftmost element of the tag is the subscript of the component which was most recently (or currently) in its critical section. The rightmost tag element refers to the component least recently in its critical section.
} 
3. Divide the remaining $n$ ? rows into $n$ groups of $(n-1)$ ! rows. In group $i, i=1, \ldots, n$, let the output state be $z_{i}$ equal to $I$ and $z_{j}$ equal to $0, j \neq i$. Give each row in group $i$ a unique tag which has $i$ in the first position and the subscripts of the other components in the other positions. (A control flow table as specified thus far is given in Table 8 for $n=3)$

4. The table entries are determined as follows:

a. In rows with all $\mathrm{z}_{i}$ equal to 0 ,

(1) there is a stable entry in the column with all input variables equal to 0 . Other columns have unstable entries.

(2) For entries which are unstable, compute a sub-order from the present tag which is the tag positions which are the subscripts on an input variable which is equal to 1 . Form a new tag using the last element of the sub-order as the first element of the new tag, the remaining elements of the sub-order as the final elements, and the remaining elements of the original tag as the middle elements (For tag 2, 1, 3, 4, the input states and new tags are shown in Table 9). The unstable entry is the number of the row with the new tag which has an output variable equal to 1. 
Table 8. Flow Table Set-Up for $n=3$

$$
\mathrm{x}_{1} \mathrm{x}_{2} \mathrm{x}_{3}
$$

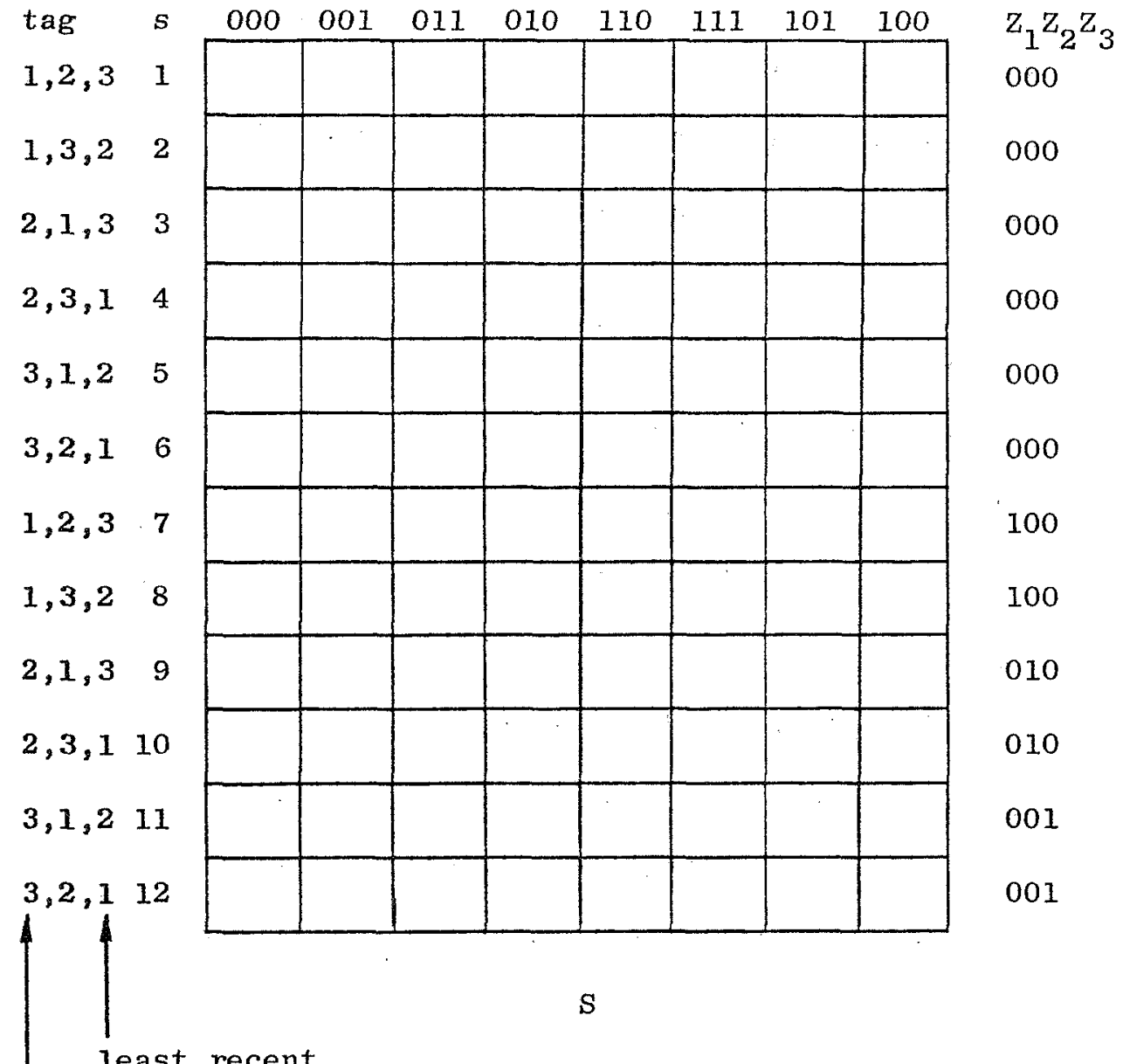

most recent 
Table 9. Tag for Next Row Given $2,1,3,4$ as Present Tag in a Row With a11 $\mathrm{z}_{i}$ Equal to 0

\begin{tabular}{|c|c|c|}
\hline $\begin{array}{l}\text { Input state } \\
\left(\mathrm{x}_{1} \mathrm{x}_{2} \mathrm{x}_{3} \mathrm{x}_{4}\right)\end{array}$ & Sub-Order & New Tag \\
\hline 0001 & 4 & $4,2,1,3$ \\
\hline 0010 & 3 & $3,2,1,4$ \\
\hline 0011 & 3,4 & $4,2,1,3$ \\
\hline 0100 & 2 & $2,1,3,4$ \\
\hline 0101 & 2,4 & $4,1,3,2$ \\
\hline 0110 & 2,3 & $3,1,4,2$ \\
\hline 0111 & $2,3,4$ & $4,1,2,3$ \\
\hline 1000 & 1 & $1,2,3,4$ \\
\hline 1001 & 1,4 & $4,2,3,1$ \\
\hline 1010 & 1,3 & $3,2,4,1$ \\
\hline 1011 & $1,3,4$ & $4,2,1,3$ \\
\hline 1100 & 2,1 & $1,3,4,2$ \\
\hline 1101 & $2,1,4$ & $4,3,2,1$ \\
\hline 1110 & $2,1,3$ & $3,4,2,1$ \\
\hline 1111 & $2,1,3,4$ & $4,2,1,3$ \\
\hline
\end{tabular}


b. In rows with exactly one output variable, say $z_{i}$, equal to 1 , the first element of the tag must be $i$.

(1) If all input variables are 0 , the new row is the row with the same tag and all output variables equal to 0 .

(2) If input variable $x_{i}$ is equal to 0 , compute a new tag as in Step 4a(2). The unstable entry is the number of the row with the new tag.

(3) If $p$ input variables are equal to $1,1 \leq p \leq n$ and $x_{i}$ is equal to 1 , find the sub-order of elements in the present tag for which the corresponding input variables have the value 1 . If the sub-order consisting of the $p-1$ elements which are not equal to $i$ is exactly the same as the sub-order consisting of the final $p-1$ elements in the present tag, the entry is a stable entry. If not, form a new tag using the sub-order corresponding to the $\mathrm{p}-1$ input variables which are $I$ as the suffix of the new tag and the remaining elements of the present tag as the prefix. The unstable entry is the number of the row with the new tag which has an output variable equal to 1 (For $\mathrm{n}=4$ and present tag $1,4,2,3$ there are stable entries in columns $x_{1} x_{2} x_{3} x_{4}=1000$, 1010, 1110, 1111. Each row has n stable entries. A complete list of the new tags for the row with tag $2,1,3,4$ and $Z_{2}$ equal to 1 is shown in Table 10 . 
Table 10. Tags for Next Row Given 2,1,3,4 as the Present Tag and $\mathrm{Z}_{2}$ Equal to 1 for the Present Row

Input state

0000

0001

0010

0011

0100

0101

0110

0111

1000

1001

1010

1011

1100

1101

1110

1111
Sub-Order

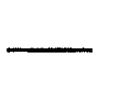

4

3

3,4

2

2,4

2,3

$2,3,4$

1

1,4

1,3

$1,3,4$

2,1

$2,1,4$

$2,1,3$

$2,1,3,4$
New Tag

$2,1,3,4$

$4,2,1,3$

$3,2,1,4$

$4,2,1,3$

$2,1,3,4$ (stable)

$2,1,3,4$ (stable)

$2,1,4,3$

$2,1,3,4$ (stable)

$1,2,3,4$

$4,2,3,1$

$3,2,4,1$

$4,2,1,3$

$2,3,4,1$

$2,3,1,4$

$2,4,1,3$

$2,1,3,4$ (stable) 
The control flow tables generated by this procedure for $n=2$ and $n=3$ are shown in Tables 11 and 12 , respectively.

The key steps in Procedure $U$ are those which determine the next row to be entered. This is always done in such a manner that the component which has been out of its critical section the longest will be enabled next, the modification of the tags in Step $4 \mathrm{a}(2)$ and $4 \mathrm{~b}(3)$ ensures that in the presence of multiple requests, the control will first enable the component which has been out of its critical section the longest and make the other components with pending requests next in line after the first component enabled executes its critical section. Procedure U satisfies conditions $1-4$ of Theorem 8 , so Restriction 1 of the mutual exclusion problem must be satisfied. The fact that Restriction 2 is also satisfied follows directly from the way the procedure determines the next component to be enabled. It follows that:

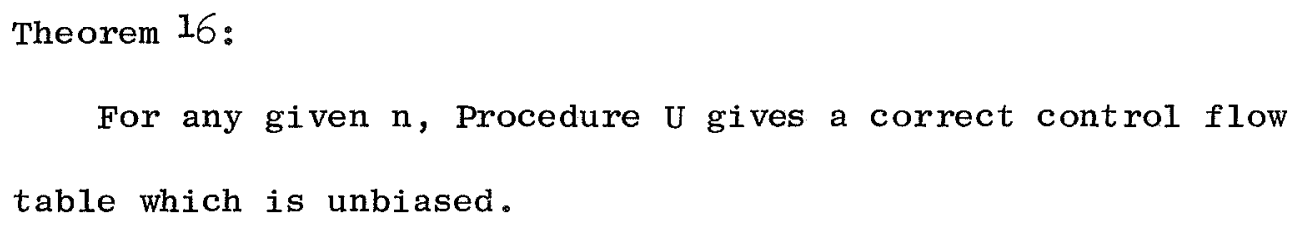

A direct consequence of the definition of an unbiased control flow table is the fact that for a given row with its past history of critical section executions (tag) and for a given input state, the flow table entry is always uniquely specified. 
Table 11. Unbiased Control Flow Table From procedure $U, n=2$

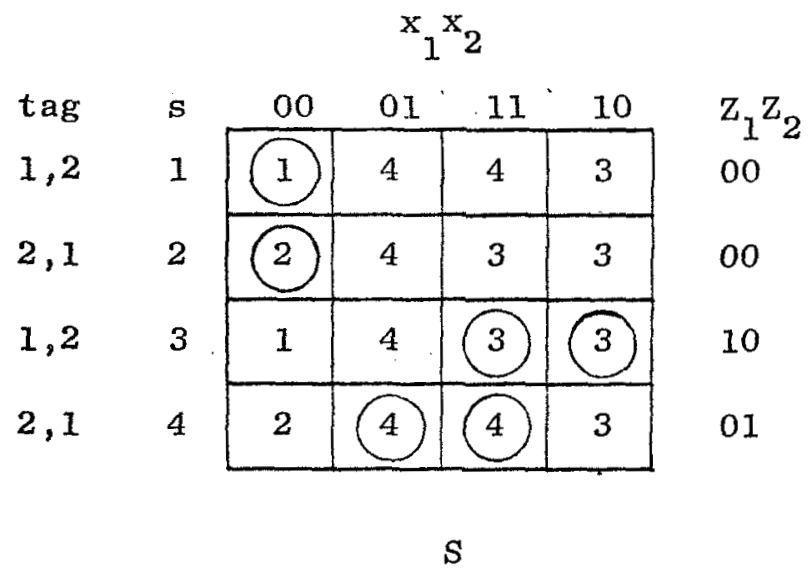


Table 12. Unbiased Control Flow Table Fron Procedure $U, n=3$

\begin{tabular}{|c|c|c|c|c|c|c|c|c|c|c|}
\hline tag & $\mathrm{s}$ & 000 & 001 & 011 & 010 & 110 & 111 & 101 & 100 & $\mathrm{Z}_{1} \mathrm{Z}_{2} \mathrm{Z}_{3}$ \\
\hline $1,2,3$ & 1 & (1) & 11 & 11 & 9 & 10 & 11 & 12 & 7 & 000 \\
\hline $1,3,2$ & 2 & (2) & 11 & 9 & 9 & 10 & 9 & 12 & 8 & 000 \\
\hline $2,1,3$ & 3 & (3) & 12 & 11 & 9 & 8 & 12 & 12 & 7 & 000 \\
\hline $2,3,1$ & 4 & (4) & 12 & 11 & 10 & 8 & 7 & 7 & 7 & 000 \\
\hline $3,1,2$ & 5 & (5) & 11 & 9 & 10 & 10 & 10 & 7 & 8 & 000 \\
\hline $3,2,1$ & 6 & (6) & 12 & 9 & 10 & 8 & 8 & 7 & 8 & 000 \\
\hline $1,2,3$ & 7 & 1 & 11 & 11 & 9 & 8. & ) & (7) & (7) & 100 \\
\hline$i, 3,2$ & 8 & 2 & 11 & 9 & 9 & (8) & 8 & 7 & (8) & 100 \\
\hline $2,1,3$ & 9 & 3 & 12 & (9) & (9) & 10 & (9) & 12 & 7 & 010 \\
\hline $2,3,1$ & 10 & 4 & 12 & 9 & (10) & (10) & (10) & 7 & 7 & 010 \\
\hline $3,1,2$ & 11 & 5 & (11) & (11) & 10 & 10 & (11) & 7 & 8 & 001 \\
\hline $3,2,1$ & 12 & 6 & (12) & 11 & 10 & 8 & (12) & (12) & 8 & 001 \\
\hline
\end{tabular}




\begin{abstract}
Theorem 17:
For each $n$, the correct control flow table that is unbiased and has $2 n$ : internal states is unique ${ }^{*}$.
\end{abstract}

CENTRAL VERSUS DISTRIBUTED CONTROL

To this point, all control and decision-making has been performed in a single component. As the number of components controlled ( $n$ ), becomes large so does the number of inputs and outputs for this component. Fortunately, however, we have shown that the number of internal states which are necessary to obtain a correct solution for each $\mathrm{n}$ is just $\mathrm{n}+1$. In this section, we will briefly discuss some alternate control organizations. Rather than concentrating all decision-making in a single control component we will consider the consequences of using more than one. Before proceeding, we must introduce some terminology which will be useful in the following discussion. The question of exactly where the "control" lies in a given system is rather difficult to specify. Thus far, for the mutual exclusion problem, we have considered the control function to reside in component $\mathrm{C}_{\mathrm{n}+1}$ of Fig. 2. This is natural because of the interpretation we attach to this system; however it is also conceivable that in some situation the control function might be thought to reside the components $C_{1}, C_{2}, \ldots, c_{n}$ which in turn drive the single component $C_{n}+1$.

* except for the numbering of the internal states. 
We will resolve such ambiguities in an arbitrary way be simply stating for a given system where the control responsibility is assumed to be. In the case of the mutual exclusion problem as shown in Fig. 2 the control is assumed to be in component $C_{n}+1$.

The parallel system in Fig. 2 is an example of a system with a central control mechanism. More precisely we say the following:

Definition:

In a given parallel system, if the control function is performed by a single component, that system is said to have central control.

If a system does not have central control, it is said to have distributed control; that is, the control function is performed by more than one component. For a given circuit it is often possible to partition the circuit in many different ways, making it difficult to determine if the circuit represents a single component or several components which communicate with each other. In the case of a parallel system, as defined in Definition 1 , we distinguish components on the basis of the delay assumptions for a parallel system. Any circuit in which it must be assumed that delays are finite and bounded in order to ensure correct operation is considered to be a single component. On the other hand, if it is possible to partition a circuit such that the parts can communicate even though the delays in the interconnecting lines are finite and unbounded, the parts are considered to be separate and distinct components. 


\section{Linear Control}

In this section, distributed control solutions to the mutual exclusion problem are considered. One type of distributed control is shown in Fig. 6. In this case each component $C_{i}, 1 \leq i \leq n$, has its own control component $\mathrm{C}_{n+i}$ with which it communicates in the same manner as components communicate with the central control in Fig. 2 . Before component $C_{i}$ can be enabled, its control component must communicate with its left and right neighbor control components to determine if it is possible for $C_{i}$ to enter its critical section. With this type of organization it is necessary to propagate a request for permission to enter a critical section to all control components. We assume each component can communicate only with its left and right neighbors. Because line delays are unbounded, when a control component produces an output value transition it must recognize an input value change produced in recognition of the propagation of its own output value before it can proceed. ${ }^{*}$ This means that pairs of lines are required, one to send a request and one to receive the reply. Furthermore a control component must not only send requests to its neighbors but receive requests from them as we11. The general form of a control component is shown in Fig. 7 .

* A further discussion of the consequences of the line delay assumption is given in [6]. 


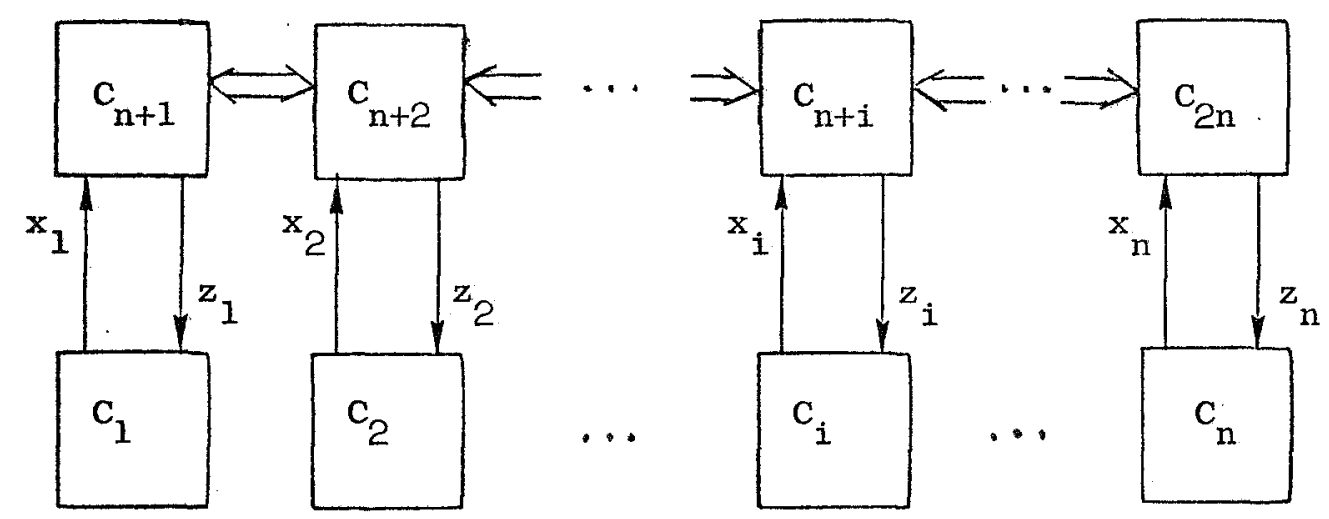

Figure 6. A linear distributed control for the mutual exclusion problem. 


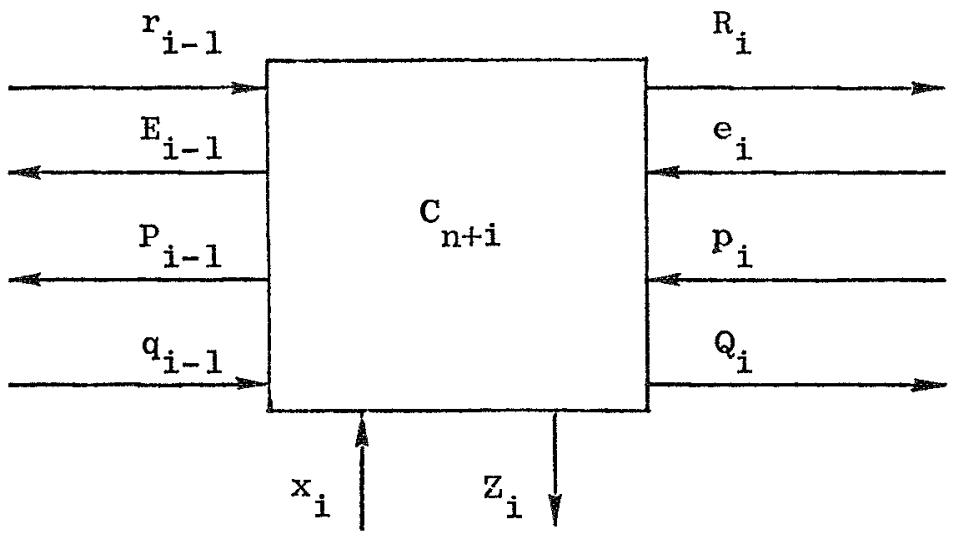

$$
\begin{aligned}
& (1 \leq i \leq n), x_{i}, z_{i} \text { as before }
\end{aligned}
$$

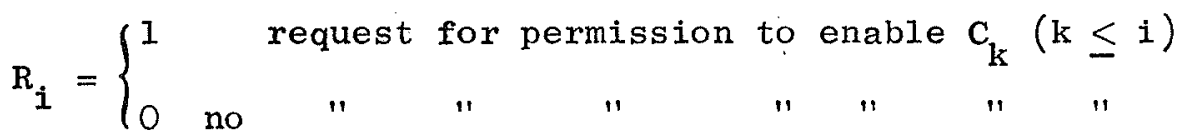

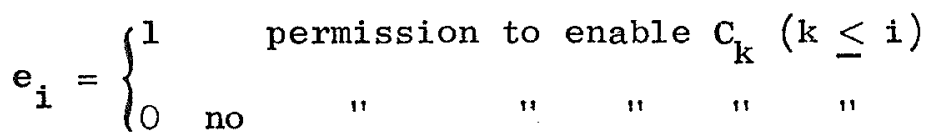

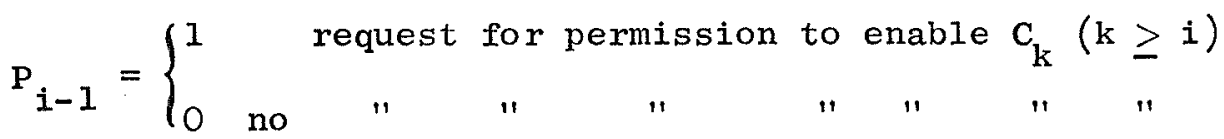

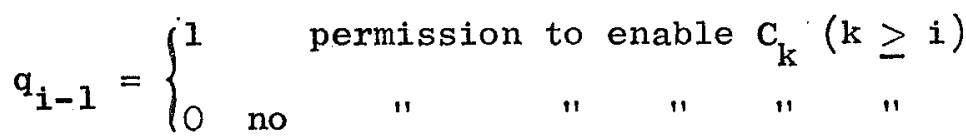

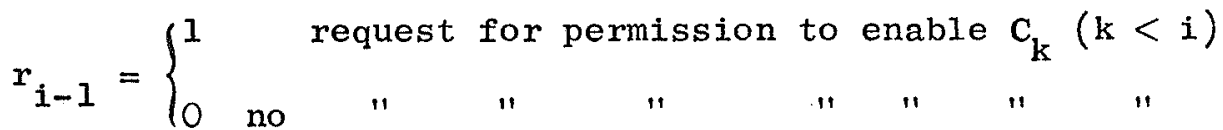

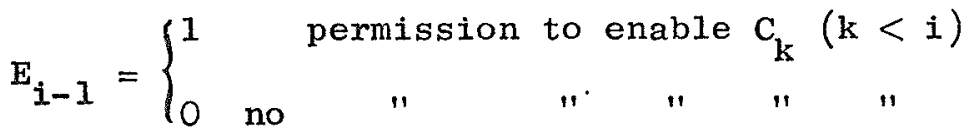

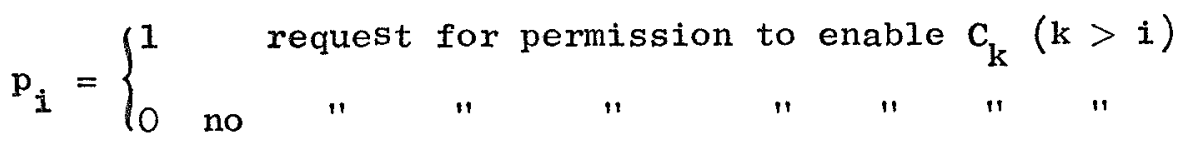

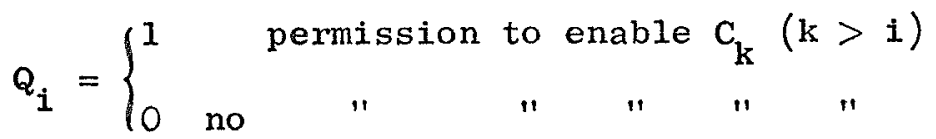

Figure 7. General form of a control component for Fig. 6. 
For correct operation, each control component must have a sufficient number of internal states to remember whether a component to his left, right, or his own component was in its critical section last. This information is necessary to resolve ties which result when multiple requests are recognized and also to know whether to pass permission to enable to another component or to wait for that component to pass permission to enable to you. Only with this information can it be guaranteed that no component is permanently excluded from its critical section. We conclude that at least three internal states are required for each general control component (the leftmost and rightmost control components need to remember only whether their own component or a neighbor was in its critical section last). The actual number of internal states is difficult to calculate and we will not do so here. However on the basis of this examination of the control structure in Fig. 6 we can conclude that the total number of internal states required for control will be at least twice as many as for a central control. Of course the number of inputs and the structure of each control component in Fig. 6 is fixed so we can add components simply by adding another control component without any redesign. Without examining actual implementations of central control components, which we do not propose to do in this paper, it is difficult to make any evaluation of either approach on a basis other than the total number of internal states required for the control function. The distributed nature of the control affects the choice of the initial internal state for each control component. That is, if each control component is started in the same 
initial state it is always possible for the system to be incorrect. Suppose each control component were initialized so that it thought its own component had been out of its critical section the longest. Then it would be possible for simultaneous requests to arrive and for each control to wait indefinitely for enabling permission from its neighbors. As a result only certain combinations of initial control component states can be used. For example if $n=4$ then we might initialize $\mathrm{C}_{5}$ to think $\mathrm{C}_{1}$ was in its critical section last and $\mathrm{C}_{6}, \mathrm{C}_{7}$, and $\mathrm{C}_{8}$ to think their left neighbor was in a critical section last. In the use of multiple requests, $\mathrm{C}_{5}$ must give permission to $\mathrm{C}_{6}$ before it waits for permission to enable $C_{1}$. The conclusion of this discussion is that there must be a certain "asymmetry" in the choice of the initial internal states for the control functions.

We-classify the form of control in Fig. 6 as linear because it is possible to arrange the components in the manner shown in the figure where each control component communicates only with its left and right neighbors [11]. Many other forms of linear control are also possible in which groups of components could communicate with a common control which would then communicate with its neighbors. The general organization. is shown in Fig. 8. It is our conjecture that in all such organizations the number of internal states for the control is always greater than the number required for a central control. Hierarchical Control

A different distributed control structure is shown in Fig. 9. This structure is know as a hierarchical structure because in this case before a component can be enabled permission must be received 


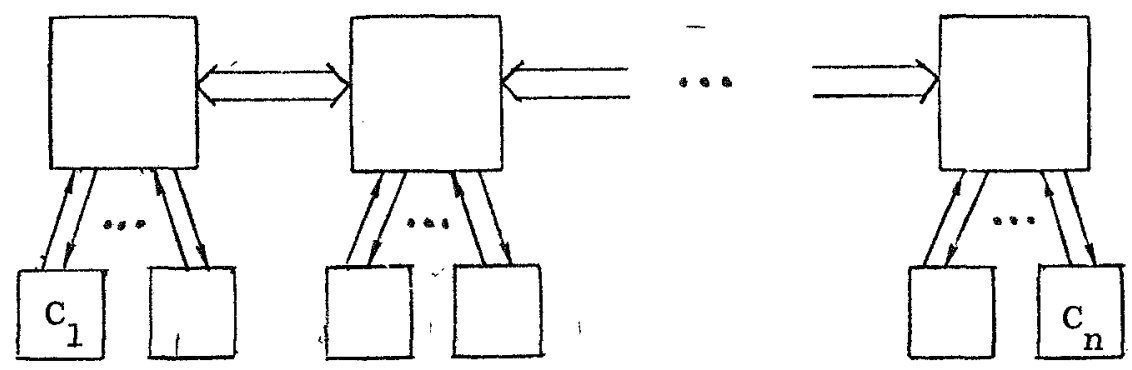

Figure 8. General organization for a linear distributed control. 

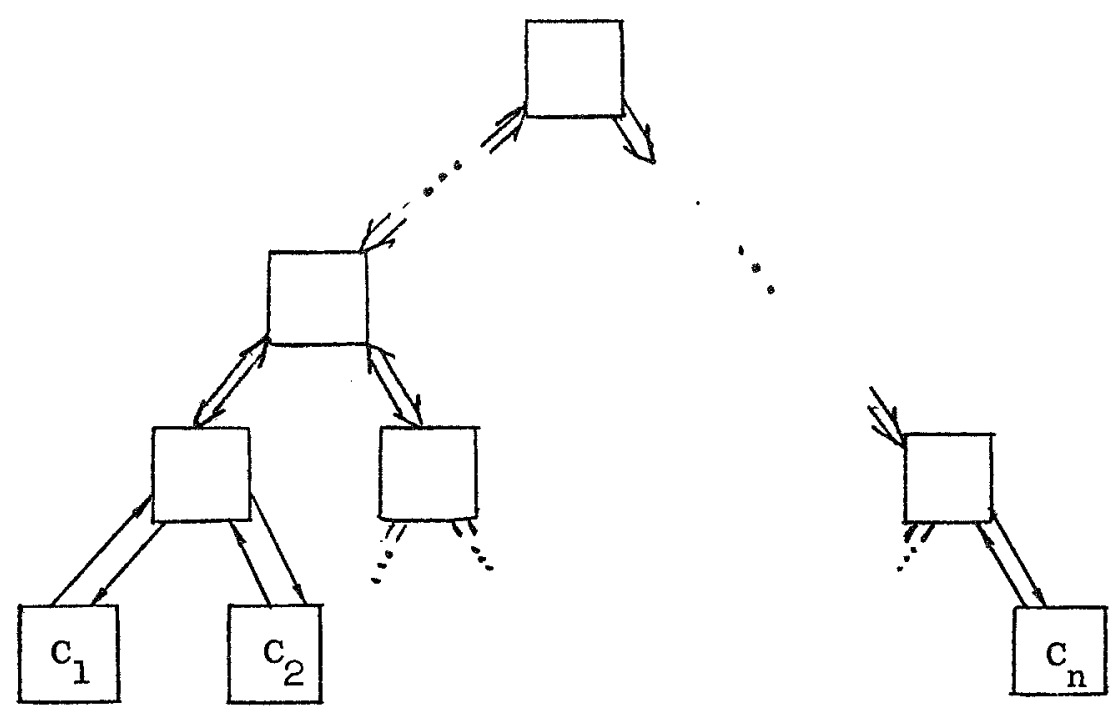

Figure 9. A binary tree hierarchical control structure. 
from the control components higher in the control "tree". Fig. 9 is an example of a binary control tree where each lower level control communicates with two components and higher level control components communicate with two lower level components. It can be shown that such a tree has $\mathrm{n}-1$ control components (non-terminal nodes) and by arguments similar to those in the last section it follows that (1) there must be an "asymmetry" in the choice of the initial control state and (2) the total number of internal states required for control components is greater than the number required for central control. We conjecture these conclusions are valid for hierarchical tree control structures which are not restricted to be binary. Both linear and hierarchical control structures are biased because the control cannot store the complete history of accesses to critical sections. 
CONCLUSIONS

The use of the flow table model has made it possible to characterize in a precise way the cost of a correct solution to the mutual exclusion problem as measured by the number of internal states required by the control function. In addition, procedures can be given to generate correct control flow tables.

Distinctions between central and distributed (linear, hierarchical) control can also be made in this model and the effects of one type of control over the other evaluated. More work needs to be done in this area. 


\section{REFERENCES}

[1] Adams, D.A. A computation model with data flow sequencing. CS-117 (Thesis), Computer Science Department, Stanford University, Stanford, California (Dec 1968).

[2] Adams, D.A. A model for parallel computations. Proc. Symp. on Parallel Processor Systems, Technologies, and Applications, Naval Postgraduate School, Monterey, Calif. June, 1969 (in press).

[3] Ashcroft, E. and Manna, Z. Formalization of properties of parallel programs. AIM-110, Artificial Intelligence Project, Stanford University, Stanford, Calif. (Feb 1970).

[4] Bredt, T.H. and McCluskey, E.J. A model for parallel computer systems. Technical Report No. 5, SEL Digital Systems Laboratory, Stanford University, Stanford, California (Apr 1970).

[5] Bredt, T.H. Analysis and synthesis of concurrent sequential programs. Technical Report No. 6, SEL Digital systems Laboratory, Stanford University, Stanford, Calif. (May 1970).

[6] Bredt, T.H. Analysis of parallel systems. Technical Report No. 7, SEL Digital Systems Laboratory, Stanford University, Stanford, Calif. (to appear).

[7] Dennis, J.B. and Van Horn, E.C. Programming semantics for multiprogrammed computations. Comm. ACM 9 (March 1966). $143-155$.

[8] Dijkstra, E.W. Solution of a problem in concurrent programming control. Comm. ACM 8 (Sept 1965), 569.

[9] Dijkstra, E.W. The structure of the "THE" multiprogramming system. Comm. ACM 11 (May 1968), 341-346.

[10] Dijkstra, E.W. Co-operating sequential processes in Programming Languages, Genuys, F. (Ed.), Academic Press New York (1968).

[11] Hennie, F.C. Finite State Models for Logical Machines. John Wiley and Sons, New York, N.Y. (1968).

[12] Karp, R.M. and Miller, R.E. Properties of a model for parallel computations: determinacy, termination, queueing. SIAM J. Appl. Math., 14 (Nov 1966), 1390-1411. 
[13] Karp, R.M, and Miller, R.E. Parallel program schemata: a mathematical model for parallel computation. IEEE Conference Record of the 8th Annual Symposium on Switching and Automata Theory (Oct 1967), 55-61.

[14] Karp, R.M. and Miller, R.E. Parallel program schemata. J. of Computer and System Sciences 3, 2 (May 1969), 147-195.

[15] Knuth, D.E. Additional comments on a problem in concurrent programming control. Comm. ACM, 9 (May 1966), 321-322.

[16] Lampson, B.W. A scheduling philosophy for multiprocessing systems. Comm. ACM 11 (May 1968), 347-360.

[17] Luconi, F.I. Completely functional asynchronous computational structures. IEEE Conference Record of the 8 th Annual Symposium on Switching and Automata Theory (Oct 1967), 62-70.

[18] Luconi, F.L. Asynchronous computational structures. MAC-TR-49 (Thesis), Massachusetts Institute of Technology, Cambridge, Massachusetts (Feb 1968).

[19] Luconi, F.L. Output functional computational structures. IEEE Conference Record of the 9th Annual Symposium on Switching and Automata Theory (Oct 1968), 76-84.

[20] Manna, Z. Termination of algorithms. Computer Science Department, Carnegie-Mellon University, Pittsburgh, Pennsylvania (Apr 1968).

[21] Manna, Z. Properties of programs and the first-order predicate calculus. J. ACM (Apr 1969).

[22] Manna, Z. The correctness of programs. J. of Computer and System Sciences, 3 (May 1969).

[23] Manna, $\mathrm{Z}$. The correctness of non-deterministic programs. Artificial Intelligence J. 1, 1 (1970).

[24] Mccluskey, E.J. Introduction to the Theory of Switching Circuits. McGraw-Hill Book Co., New York, N.Y. (1965).

[25] Muller, D.E. and Bartky, W.S. A theory of asynchronous circuits. Proc. of an International Symposium on the Theory of Switching, the Annals of the Computation Laboratory of Harvard University, Vo1. 29, Part I, Harvard University Press (1959), 204-243. 
[26] Rodriquez, J.E. A graph model for parallel computations. Ph.D Thesis, MIT, Department of Electrical Engineering, Cambridge, Massachusetts (Sept 1967).

[27] Slutz, D.R. The flow graph schemata model of parallel computation. MAC-TR-5I (Thesis), MIT, Cambridge, Massachusetts (Sept 1968). 
Unclassified

Security Classification

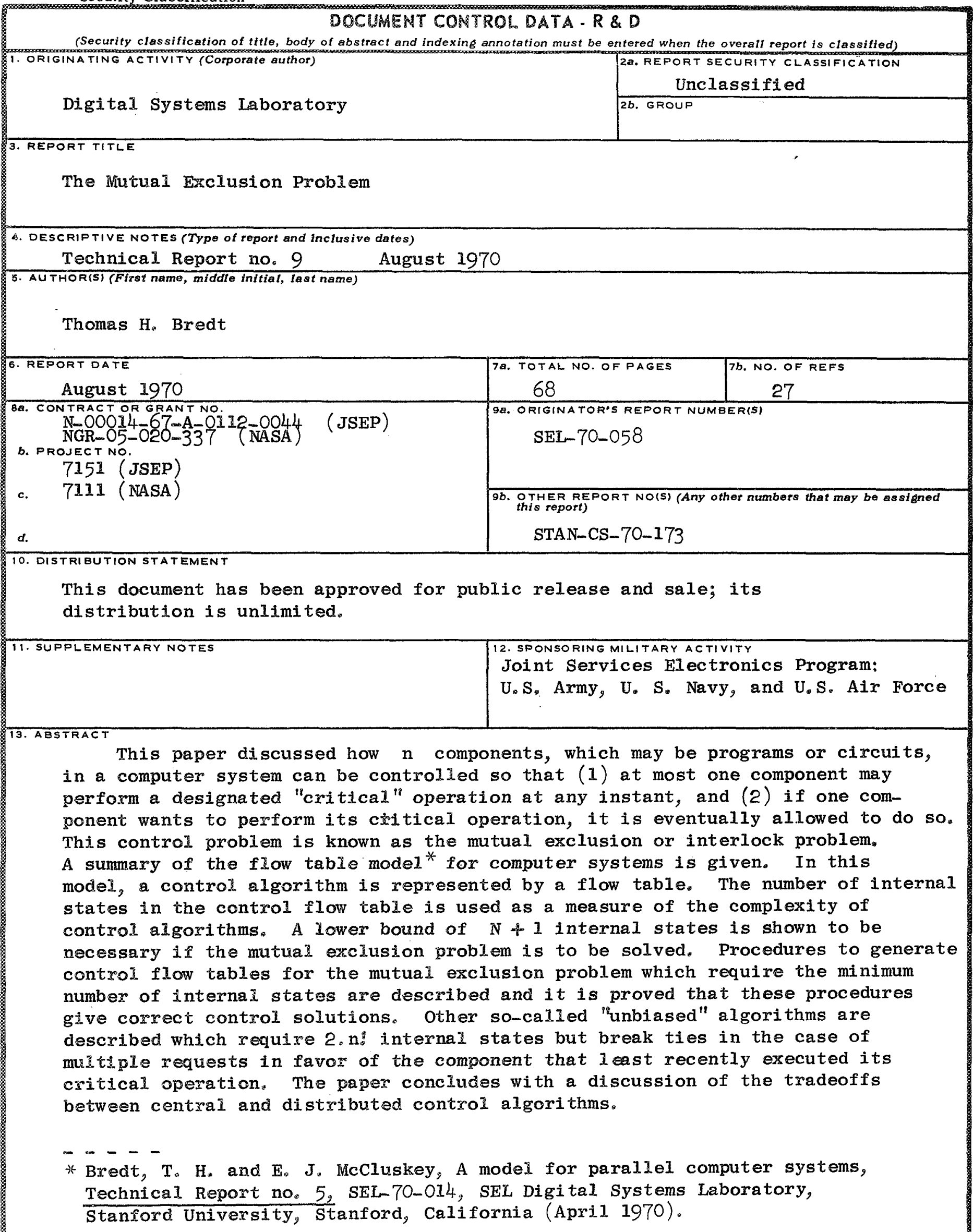




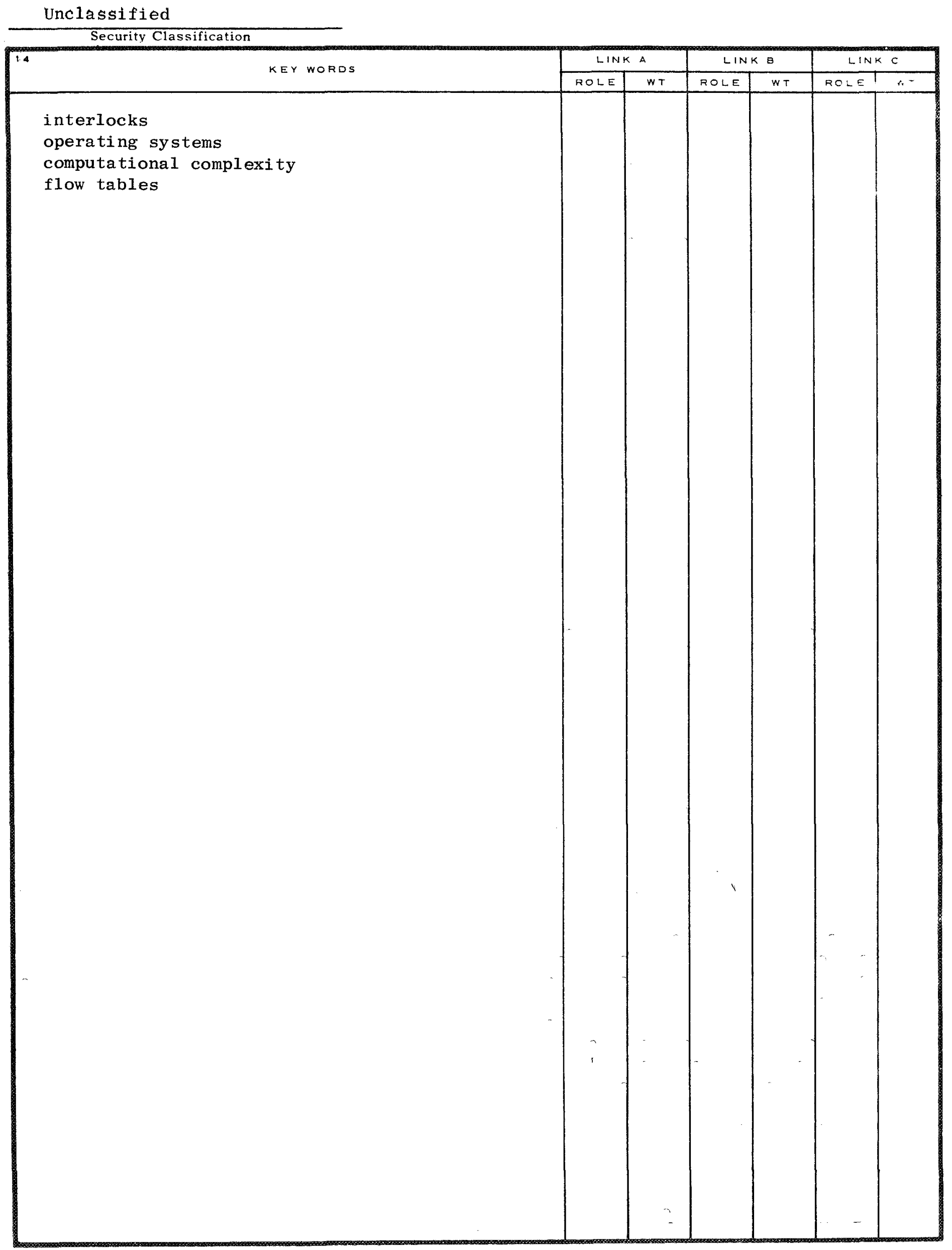

Security Classification 